UCRL-ID-127438

\title{
The Content and Structure of MCF Files
}

\author{
G. W. Hedstrom \\ L. J. Cox \\ S. T. Perkins
}

May 6, 1997

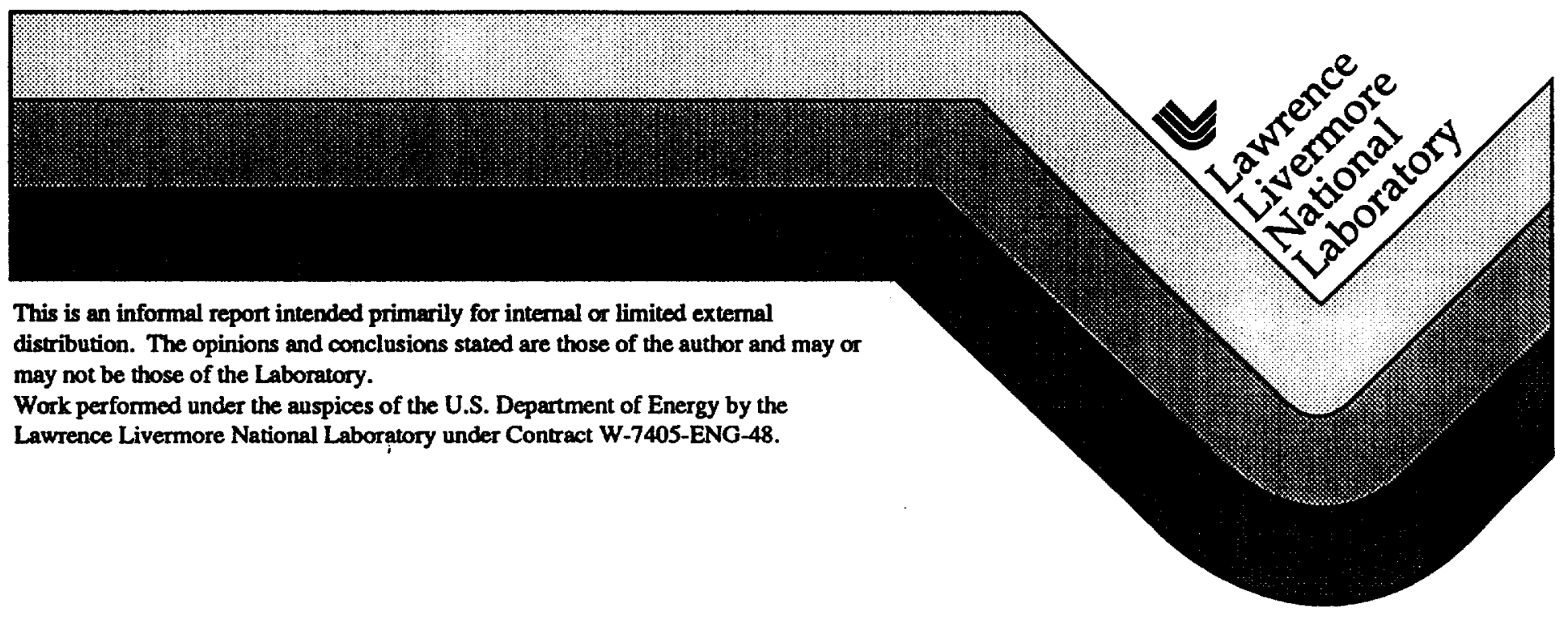




\section{DISCLAIMER}

This document was prepared as an account of work sponsored by an agency of the United States Government. Neither the United States Government nor the University of California nor any of their employees, makes any warranty, express or implied, or assumes any legal liability or responsibility for the accuracy, completeness, or usefulness of any information, apparatus, product, or process disclosed, or represents that its use would not infringe privately owned rights. Reference herein to any specific commercial product, process, or service by trade name, trademark, manufacturer, or otherwise, does not necessarily constitute or imply its endorsement, recommendation, or favoring by the United States Government or the University of California. The views and opinions of authors expressed herein do not necessarily state or reflect those of the United States Government or the University of California, and shall not be used for advertising or product endorsement purposes.

This report has been reproduced directly from the best available copy.

Available to DOE and DOE contractors from the Office of Scientific and Technical Information

P.O. Box 62, Oak Ridge, TN 37831

Prices available from (615) 576-8401, FTS 626-8401

Available to the public from the

National Technical Information Service

U.S. Department of Commerce

5285 Port Royal Rd.,

Springfield, VA 22161 


\title{
The Content and Structure of MCF Files
}

\author{
G. W. Hedstrom, L. J. Cox, and S. T. Perkins
}

6 May 1997 


\section{Contents}

Overview 3

The purpose of mcfgen. . . . . . . . . . . . 3

The history of mcfgen and mcfbin. ........... 4

Locations of mcfgen and mcfbin ............. 4

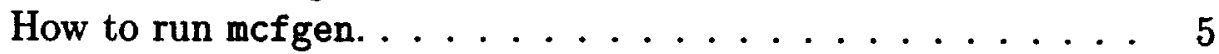

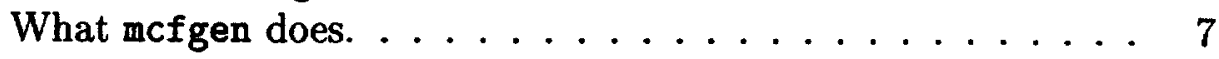

The setup. ....................... 7

Initial processing for each target. . . . . . . . 8

Loop over the reactions . . . . . . . . . . 9

Library type 1 grid-based data .......... . . 10

Library type 0 : data by energy group . . . . . . . . 10

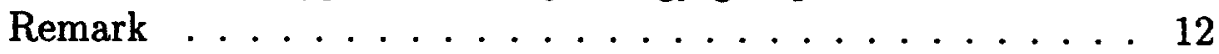

Portability issues .................. 12

Various identifiers $\mid \quad 14$

The code for particles . . . . . . . . . . . . . 14

The C Reaction Identifiers . . . . . . . . . . . . 16

The S Reaction Switch . . . . . . . . . . . . . 19

The I_number identifier of the kinds of data . . . . . . . 21

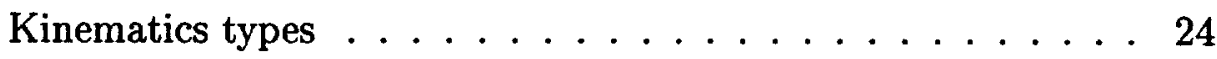

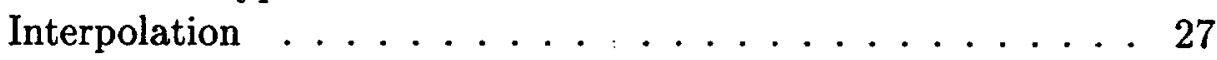

File formats $\quad 28$

The file 'mcfgen.input' . . . . . . . . . . . . . 28

The format of the 'library' file . . . . . . . . . . 32

Background information ........... 32

The overall directory ............ 33

The target directory ............ 34

The format for 'mcf.asc' .............. 40

The format for 'mcf.bin' .............. 53

The segments of a kinematics directory. . . . . . . . 54

The kinematics directories ........... . 57

Reaction directories. ............ 61 
Target directory. . . . . . . . . . . . 63

Overall directory .............. 64

Code details for mcfgen $\quad 67$

Structures identifying the data . . . . . . . . . 67

The array i_react_data . . . . . . . . . 67

The array r_react_data ........... 68

The array ident_data . . . . . . . . . 69

Equiprobable bins . . . . . . . . . . . . . 70

Correlated angle-energy equidistribution . . . . 72

Log-log interpolation . . . . . . . . . . . . . 74

$\begin{array}{lr}\text { References } & 82\end{array}$ 


\section{Overview}

The Nuclear Data Group at Lawrence Livermore National Laboratory maintains libraries of data for the reactions of various incident particles on a wide selection of target nuclei. Cross sections and available energy are always given, but in addition there may be angular distributions, energy distributions, and multiplicity of ejected particles. For use in all-particle Monte Carlo codes, this data is available as Cray binary files, mcf $1, \ldots, \operatorname{mcf} 7$, one file for each incident particle: neutron, proton, deuteron, triton, ${ }^{3} \mathrm{He}$, alpha, and photon.

The purpose of this document is to explain the data processing and to describe the format of the files. The creation of the binary files is done in two steps. The code mcfgen is the first step, and it does the processing of the library data. The output of mcfgen is an ASCII file 'mcf.asc', and this file serves as input to the code mcfbin, which produces a corresponding binary file 'mcf .bin'. The mcfbin merely changes the order of the data and converts it to binary format. The formats of the 'mcf.asc'? and 'mcf.bin' files are given later in this document.

This document is organized as follows. This first chapter gives a summary of what mcfgen does, including a description of the processing the code does for each type of data. The second chapter is a reference, providing lists of the meanings of the various identifiers used. Chapter 3 gives descriptions of the contents and formats of the input and output files. The final chapter is for the benefit of the programmer, and it provides details about the data structures and numerical algorithms used.

:

\section{The purpose of mcfgen.}

The library data is given in terms of pairs of numbers, such as (energy of the incident particle, cross section) or (cosine of the angle for the ejected particle, probability). Two types of processing are done. For some of the data, including the cross sections, mcfgen calculates the average cross section for incident particles in a list of predefined energy groups. For probabilitydistribution data mefgen computes equiprobable bins.

Each run of mcfgen is for a specific incident particle: neutron, pro- 
ton, deuteron, triton, ${ }^{3} \mathrm{He}$, alpha, or photon. Therefore, in order to create 'mcf.bin' files for all of these incident particles, we have to make several runs of mcfgen and mcfbin.

\section{The history of mcfgen and mcfbin.}

The use of Monte Carlo methods for radiation transport at LLNL started with only neutrons and photons, and the code to do these calculations was tart, maintained by E. F. Plechaty. The library reaction data for tart was maintained by R. J. Howerton, and it was kept in binary files 'endl' (for neutron data) and 'egdl' (for gamma-ray data). R. E. Dye maintained the codes clyde and trtl to process this data to produce input files for tart, and documentation may be found in the omega manual [2].

In the early 1980s all of the data-processing codes, including clyde and trt1, were merged into one big code omega [2]. Since then, the Code Group has dismantled omega into its components for a couple of reasons. For one thing, as computers and operating systems changed, it was éasier to update several small codes, one at a time, than to change omega all at once and make sure that it was reliable. For another thing, J. A. Rathkopf wrote a Monte Carlo code mcapm to keep track of several light-weight particles: neutron, proton, deuteron, triton, ${ }^{3} \mathrm{He}$, alpha, and gamma. Even though the data processing for mcapm had much in common with that required for tart, it was easier to do this with a new code newct than to fit this processing into omega.

The current version of mcfgen is derived from newct. The most significant changes to the code are that we have patched a number of memory leaks, and we added documentation. In many places we substituted clearer coding.

\section{Locations of mcfgen and mcfbin}

The standard version of mcfgen is located in the directory

$$
\text { /nds/bin }
$$

on the Nuclear Data Group's computer network. The source code for the program mcfgen on the Nuclear Data Group's computer network is located 
in the directory

$$
\text { /nds/processing/mcf/mcfgen }
$$

and it is under the control of the sccs file-management system.

The source code for mcfbin is located in the directory

$$
\text { /nds/processing/mcf/mcfbin }
$$

The Fortran code is machine-dependent for a few of the subroutines in this directory. These routines are distinguished by the presence of the letters 'CRY' or 'SUN' in the file name. Correspondingly, this directory also contains two versions of Makefile, namely, MakefileCRY and MakefileSUN. It is the convention to use Cray binary mcf files. Consequently, even though the source files for mcfbin are maintained on the network of Sun computers, the code is actually run on one of the Crays.

\section{How to run mcfgen.}

We shall give most of our attention to mcfgen because that is where the data processing is done. One may run mcfgen alone, but becaúse it is just one step in a chain, one should also examine the script for the entire process, including creation of the 'library' files described below,

$$
\text { /nds/bin/build.mcf.general }
$$

For mcfbin there are two scripts,

$$
\text { /nds/processing/scripts/tar.mcfbin }
$$

and

$$
\text { /nds/processing/scripts/build.mcfbin }
$$

The script tar.mcfbin assembles the source code for mcfbin into one subdirectory along with the 'mcf.asc' files, and it produces a compressed file mcfbin.tar.gz for transfer to a Cray computer. The script build.mcfbin uncompresses this file, makes the code mcfbin, and produces the 'mcf.bin' files, renaming them mcf $1, \ldots$, mcf 7 .

If one wants to run the code mcfgen alone, the current directory must contain the following files or links to them. 
'bdfls': An ASCII file containing group boundaries, flux weightings, atomic masses, half-lives, physical constants, temperature sets, and atomic subshell designators. The standard instance of this file on the Nuclear Data Group's computer network is

$$
\text { /nds/processing/constants/physcons }
$$

' '1ibrary': A binary file of library nuclear data. This file is made by running the codes create and endlret. See the script build.mcf.general.

'mcfgen.input': A file in which the user may specify various data, the most important of which are the identity of the incident particle and the range of targets. The standard 'mcfgen.input' files are located on the Nuclear Data Group's computer network as

$$
\text { /nds/processing/inputs/mcfgen.input.N }
$$

where the $\mathrm{N}$ is one of the values 1 (neutron), 2 (proton), 3 (deuteron), 4 (triton), 5 ( ${ }^{3} \mathrm{He}$ ), 6 (alpha), or 7 (photon). These files are very rigid in format, and the spacing must be exact. For example, the standard input file for a proton is

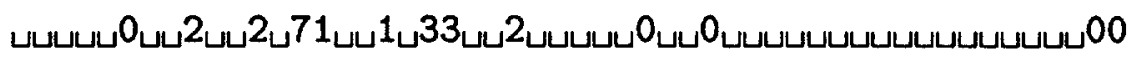

பบ1001 8016

Here $\sqcup$ marks a space. The significance of these numbers is explained in the chapter on files, so we point out only the most important ones here. The first 0 indicates that the file has a second line containing the range of targets. The first 2 specifies that the incident particle is a proton. The last zero before the 00 specifies that, except for the angular distributions and the energy distributions, the information in the output file 'mcf .asc' consists of averages over energy groups. The case of an incident photon has a 1 in that location, indicating that this data is given as grid-based values. The second line identifies the targets in terms of atomic number $Z$ and mass number $A$ using the code $1000 * Z+A$. Thus, the targets range from isotopes ${ }^{1} \mathrm{H}$ through ${ }^{16} \mathrm{O}$. 
The standard way to run the code is simply to type

$$
\text { mcfgen }
$$

in a directory containing soft links for the files 'bdfls', 'library', and 'mcfgen.input'. There is a variant to permit the user to process the data for a single target without changing the 'mcfgen.input' file. In particular, one may use the command line to override the range of targets given in the 'mcfgen.input' file by typing

mcfgen nnza

Here, nnza is an integer of the form $1000 * Z+A$, coding the atomic number and mass number of the (single) target. Thus, to process only the data for protons incident on deuterium with the 'mcfgen.input' file as above, one types

mcfgen 1002

\section{What mcfgen does.}

We give here a brief overview of mcfgen. The code first does some initialization. Then it goes through the list of targets. For each target the code reads a list of reactions. For each reaction it reads data from the 'library' file, processes the data, and writes the results to the 'mcf.asc' output file.

\section{The setup.}

In the initialization procedure the following subroutines are called.

init: Open the input files and create the 'mcf.asc' file. A file for warning messages, 'mcfgen.log', is also created. This subroutine also reads basic data from the 'bdfls' file, including some physical constants and the identities of the targets.

getdate: Get and store the date of the run for later printing to 'mcf .asc'. The format is 'yymmdd', so that on 22 May 1995 the number 950522 is returned. 
libhdr: Read the basic information about the 'library' file. The format of this file is given in the chapter on files.

input: Read the 'mcfgen.input' file to get the identity of the incident particle and the range of targets. This subroutine also obtains the energy group structure and the flux as a function of energy. The default flux is energy-independent. For details on how to set up a special physics problem, see the section on 'mcfgen.input' in the chapter on files.

flxav: If the data are bin averages, this subroutine computes the total flux over each energy group $g$,

$$
\phi_{g}=\int_{g} \phi(E) d E .
$$

The reason for wanting these values is that the group averages computed later are all weighted by the flux.

\section{Initial processing for each target.}

For each target the code starts with checks for consistency. It then calls the following subroutines.

trghdr: The first object of this subroutine is to read the identity and basic properties of the target. But the primary purpose is to read the array nhead, containing pointers to the data in the 'library' file. See the section on the ' 1 ibrary' file for a full description of the contents of this structure.

trgdat: This subroutine merely reads the data for a given target from the 'library' file and writes it to a scratch file, 'endata'.

rct: Here, we get the list of reactions to be processed for this target. We also identify the types of data given for these reactions. This information is transferred to local data structures as described in the chapter on Structures identifying the data. The routine rct also calls parts to get the effective Q-value for each reaction, based on the difference in masses. 
bdfmass: From a table get the target mass in atomic mass units.

bdfhalf: From a table get the halflife of the target.

santc: Eliminate from the list of reactions those which are not of interest for this run. Also write to the 'mcf .asc' output file the basic information for this target.

In main: If this is the first target, write the boundaries of the energy groups to be used in the Monte Carlo computations. The code also writes the target temperatures, but there is only one temperature because Doppler broadening is done outside of mcfgen.

models: This subroutine sets the kinematics types for the reactions. For the codes see the section for 'Kinematics' in the chapter on Various Identifiers. This routine also sets the number of equal-probability bins for the distributions of angles and energies of secondary particles.

isoprd: Make the list of secondary particles and residual nucleus for each of the reactions for this target. Note that this routine makes substitutions for unstable residual nuclei, and messages are written to the 'output' file when this is done. In particular, for a neutron incident on ${ }^{9} \mathrm{Be}$ $(\mathrm{ZA}=4009)$, the following substitutions are made.

For $\mathrm{C}=40$ substitute 3007 for 3009 .

For $\mathrm{C}=41$ substitute 3007 for 3008 .

For $\mathrm{C}=45$ substitute 2004 for 2006 .

For $\mathrm{C}=46$ substitute 4009 for 4010 .

\section{Loop over the reactions}

We have seen that for each target the routine trgdat transfers the corresponding data from the ' 1 ibrary' file to the scratch file 'endata'. The code mcfgen reads data from the file 'endata', processes it, and prints the results to the output file 'mcf.asc'. There are two very different kinds of processing done, depending on the library type. 


\section{Library type 1: grid-based data}

This type of data applies only to incident photons, and the method of interpolation is log-log. The first processing that is done is to insert enough intermediate points, so that linear-linear interpolation is accurate to within a relative error of 0.001 . This insertion is done by the routines thicken and $\log \log$. For a detailed description of this process see the section on 'Log-log interpolation' in the chapter, Code details. All of the thickened data is simply written to the 'mcf.asc' file.

The only other processing that is done with this data is that for energy distribution to a secondary particle (I_number $=4$ ). The thickened values are replaced by equally probable bins, just as for Inumber $=4$ data from Library type 0 below.

\section{Library type 0: data by energy group}

For a given reaction the order of the processing is according to the data type as given by the identifier I number. For the different values of I number the various types of data are as follows. Note that no single reaction has all of this data.

I_number $=0:$ The original data in the 'library' file is an array of pairs (incident energy, reaction cross section). The subroutine iequo computes and prints flux-weighted averages over the groups $g$,

$$
\sigma_{g}=\frac{\int_{g} \sigma(E) \phi(E) d E}{\int_{g} \phi(E) d E} .
$$

The integration is done by the trapezoid rule. The code also calculates and prints out the energy deposited and the energy available.

Inumber $=1$ : The data consists of pairs (energy deposited to secondary particle, probability) for a sequence of incident energies and cosines of the collision angle. Depending on the kinematics type, this data may be in the laboratory or the center-of-mass system. The subroutine iequ1 computes boundaries for equiprobable cosine bins, and it prints them. See the section on 'Equidistribution' in the chapter on Code details. 
Inumber $=3:$ The data consists of pairs (energy of the secondary particle, probability) for a sequence of incident energies and cosines of the collision angle. This data is in the center-of-mass system. The subroutine iequ3 computes boundaries for joint equiprobable cosine bins and energy bins, and it prints them. This type of data is given only for ${ }^{9} \mathrm{Be}$ and for the Production Cross Section Library. Note that this block of data is not self-sufficient-the angular-distribution data (I_number $=1$ ) must be present and must be consistent.

I number $=4:$ The data consists of pairs (energy of secondary, probability) for a sequence of incident energies. This data is in the laboratory system. The subroutine iequ 4 computes equiprobable energy bins and writes them (along with the incident energies) to the 'mcf . asc' output file.

Inumber $=7$ or $9:$ The cases of data type 7 and 9 are treated together by the subroutine iequ7or9. In both cases the data is an array of pairs (incident energy, multiplicity). For data type 7 the particles are fission neutrons, and for type 9 the data gives the multiplicity of emitted photons. The fission neutrons may be prompt or delayed. The code computes average multiplicities over the energy groups $g$, weighted by the reaction cross section,

$$
\bar{\nu}_{g}=\frac{\int_{g} \bar{\nu}(E) \sigma(E) \phi(E) d E}{\int_{g} \sigma(E) \phi(E) d E},
$$

It then prints the results to the 'mcf.asc' file.

Inumber $=10$ : The data in the ' 1 ibrary' file consists of pairs (energy, energy deposition). This data may be in the center-of-mass system or the laboratory system, depending on the kinematics type of the reaction. The subroutine iequ10 computes the average energy deposited in each energy group $g$,

$$
\tilde{E}_{g}=\frac{\int_{g} \tilde{E}(E) \sigma(E) \phi(E) d E}{\int_{g} \sigma(E) \phi(E) d E},
$$

and it prints the results to the 'mcf.asc' file. 
I.number $=12:$ This flag is used only for making Production Cross Section files. When $\mathrm{C}=5$ the 'library' file contains pairs $(E, Q)$, where $E$ is the incident energy and $Q$ the energy available from non-elastic reactions. The code computes and prints the group averages

$$
Q_{g}=\frac{\int_{g} Q(E) \sigma(E) \phi(E) d E}{\int_{g} \sigma(E) \phi(E) d E}
$$

This processing is done inside the subroutine iequo.

\section{Remark}

The ' 1 ibrary' file contains data for energy deposition to the residual nucleus, and mcfgen does not use this information. This data is identified by Inumber $=11$. It would be very easy to get this data out as group-averaged energy deposition. All that is needed is to modify the array irlst of permissible values of I_number in the file 'datcom. $h$ ' and to change the reading of irlst in main. In particular, in 'datcom. $h$ ' change irlst from

$$
0,1,3,4,7,8,9,10,941,942,-1
$$

to

$$
0,1,3,4,7,9,10,11,941,942,-1
$$

(The value $I$ number $=8$, indicating histogram data, is not used.) A corresponding change is needed in main, namely, the replacement of

· ELSEIF (I_number .EQ. 10)

by

ELSEIF ((I_number .EQ. 10) .OR. (I_number .EQ. 11))

\section{Portability issues}

The principal difficulty in moving mcfgen from one machine to another is that the code was originally written under the assumption that real numbers and integers occupy the same amount of storage. This assumption is now 
made only for the binary file 'library'. Accommodation of different storage lengths is made by setting the parameter INTS_REALS in the file 'machdep. $h$ '. The value of INTS_REALS is to be set equal to

$$
\text { sizeof(REAL)/sizeof(INTEGER). }
$$

Thus, on a Cray INTS_REALS $=1$ and on a Sun INTS_REALS $=2$.

The other portability issues for mcfgen relate to machine precision for REAL numbers. Two accuracy parameters, relerror and eps_ehat, are set in the file 'machdep. $h$ '. The value of relerror is used by the routine loglog, and eps_ehat is used by the routine getehat. Beware also that the entire implementation of the routine etrnc depends on the machine precision. This routine is used to test for near equality of reals.

The code mcfbin is still written with the assumption that integers and real numbers required the same amount of storage. This is so far not a problem, because mcfbin is intended only for the Cray. (We mentioned earlier that a Sun version of this code is available, but it uses a compiler option to enforce the equal-storage assumption.) 


\section{Various identifiers}

This chapter contains the following tables: the code for the particles, the reaction identifiers $C$ and $S$, the identifiers Innumber for the type of data, the kinematics types, and the interpolation code.

\section{The code for particles}

The code for identifying particles is as follows.

0: Not applicable or no secondary particle.

1: Neutron.

2: Proton.

3: Deuteron.

4: Triton.

5: ${ }^{3} \mathrm{He}$.

6: Alpha.

7: Photon.

8: Positron.

9: Electron (negative).

10: Electron.

11: Neutron as recoil nucleus.

12: Proton as recoil nucleus.

13: Deuteron as recoil nucleus.

14: Triton as recoil nucleus.

15: ${ }^{3} \mathrm{He}$ as recoil nucleus. 
16: Alpha as recoil nucleus.

18: Positron as recoil nucleus.

19: Electron (negative) as recoil nucleus. 


\section{The C Reaction Identifiers}

In the following table $y_{i}$ denotes the incident particle.

\section{Miscellaneous}

1-4: Unassigned.

5: Production cross sections.

6: Unassigned.

7: Elastic transport.

8: Large-angle Coulomb scattering.

9: Nuclear elastic scattering plus interference.

10: Elastic scattering.

\section{Emission of neutrons and gammas}

11: $\left(y_{i}, n^{\prime} \gamma\right)$ reactions.

12: $\left(y_{i}, 2 n \gamma\right)$ reactions.

13: $\left(y_{i}, 3 n \gamma\right)$ reactions.

14: $\left(y_{i}, 4 n \gamma\right)$ reactions.

15: Total fission.

16-19: Unassigned.

Emission of neutrons, charged particles, and gammas

20: $\left(y_{i}, n^{\prime} p \gamma\right)$ reactions.

21: $\left(y_{i}, p n^{\prime} \gamma\right)$ reactions.

22: $\left(y_{i}, n^{\prime} d \gamma\right)$ reactions.

23: $\left(y_{i}, n^{\prime} d \alpha \gamma\right)$ reactions. 
24: $\left(y_{i}, n^{\prime} t \gamma\right)$ reactions.

25: $\left(y_{i}, n^{\prime 3} \mathrm{He} \gamma\right)$ reactions.

26: $\left(y_{i}, n^{\prime} \alpha \gamma\right)$ reactions.

27: $\left(y_{i}, n^{\prime} \gamma 2 \alpha\right)$ reactions.

28: $\left(y_{i}, n^{\prime} t \alpha \gamma\right)$ reactions.

29: $\left(y_{i}, 2 n p \gamma\right)$ reactions.

30: $\left(y_{i}, \gamma n \alpha\right)$ reactions.

31: $\left(y_{i}, 2 n p \alpha \gamma\right)$ reactions.

32: $\left(y_{i}, 2 n d \gamma\right)$ reactions.

33: $\left(y_{i}, 2 n \alpha \gamma\right)$ reactions.

34: $\left(y_{i}, n p \alpha \gamma\right)$ reactions.

35: $\left(y_{i}, d n \gamma\right)$ reactions.

36: Unassigned.

Emission of charged particles and/or gammas

37: $\left(y_{i}, 2 \alpha \gamma\right)$ reactions.

38: $\left(y_{i},{ }^{3} \mathrm{He} \alpha \gamma\right)$ reactions.

39: $\left(y_{i}, p t \gamma\right)$ reactions.

40: $\left(y_{i}, p \gamma\right)$ reactions.

41: $\left(y_{i}, d \gamma\right)$ reactions.

42: $\left(y_{i}, t \gamma\right)$ reactions.

43: $\left(y_{i}, t \alpha \gamma\right)$ reactions.

44: $\left(y_{i},{ }^{3} \mathrm{He} \gamma\right)$ reactions. 
45: $\left(y_{i}, \alpha \gamma\right)$ reactions.

46: $\left(y_{i}, \gamma\right)$ reactions.

47: $\left(y_{i}, d \alpha \gamma\right)$ reactions.

48: $\left(y_{i}, p \alpha \gamma\right)$ reactions.

49: $\left(y_{i}, p 2 \alpha \gamma\right)$ reactions.

\section{Production of charged particle, gamma, and nucleus}

50: $\left(y_{i}, X p\right)$ reactions.

51: $\left(y_{i}, X d\right)$ reactions.

52: $\left(y_{i}, X t\right)$ reactions.

53: $\left(y_{i}, X^{3} \mathrm{He}\right)$ reactions.

54: $\left(y_{i}, X \alpha\right)$ reactions.

55: $\left(y_{i}, X \gamma\right)$ reactions.

56: $\left(y_{i}, X n\right)$ reactions.

57: $\left(y_{i}, X \beta^{-}\right)$reactions.

58-64: Unassigned.

65: Activation.

66: Reaction yielding a target with atomic number $Z_{2}$ and mass number $A_{2}$. 67-70: Unassigned.

\section{Photon interaction}

71: Coherent scattering.

72: Incoherent scattering.

73: Photoelectric interactions. 
74: Pair production.

75: Triplet production.

76-80: Unassigned.

\section{Electron interaction}

81: Ionization.

82: Bremsstrahlung.

83: Excitation.

84-90: Unassigned.

\section{Atomic paramters}

91: Subshell parameters.

92: Transition parameters.

93: Whole-atom parameters.

94-99: Unassigned.

\section{The S Reaction Switch}

The S switch is one of the parameters in the structure nhead in the directory of the 'library' file, and its purpose is the tell the significance of some other parameters in nhead, namely, $X_{1}, X_{2}, X_{3}$, and $X_{4}$. For an explanation of nhead see the 'Library' section of the chapter on Input and output.

0: There is no $X$ data.

1: The parameter $X_{1}$ is the excitation level in Mev.

2: These are pre-equilibrium and unresolved direct-interaction processes, and there is no $X$ data. Note for example, that the 'endl' library has two separate ${ }^{235} \mathrm{U}(\mathrm{n}, \gamma)$ reactions $(\mathrm{C}=46)$, a cluster reaction with $\mathrm{S}=0$ and a direct-interaction process with $\mathrm{S}=2$. 
3: We have gamma-ray production, and $X_{1}$ is the energy of the emitted photon in Mev.

5: This is an activation process, $X_{1}=1000 * Z+A$ to identify the atomic number and mass number of the residual, $X_{2}$ is the excitation level of the residual in Mev, and $X_{3}$ is its halflife in seconds.

7: We have delayed fission groups, and $X_{1}$ is the halflife of this group. But if $X_{1}=0$, then the data is a sum over all delayed groups.

8: This is a time-sequential cluster model, and $X_{1}$ gives the excitation level in Mev.

91: In this case $X_{1}$ designates the subshell. 


\section{The I_number identifier of the kinds of data}

The reaction data identifier, I_number, tells what data is stored, as given by the following table.

0: This type of data contains pairs (energy, cross section). The code mcfgen computes and prints the averages over the energy groups $g$ weighted by the flux $\phi$,

$$
\sigma_{g}=\frac{\int_{g} \sigma(E) \phi(E) d E}{\int_{g} \phi(E) d E} .
$$

The flux is usually constant, but other choices may be specified by the 'mcfgen.input' file.

1: The data consists of pairs (cosine of the collision angle, probability) for a sequence of incident energies. This data may be in the center-of-mass system or the laboratory system, depending on the kinematics type of the reaction. The code mcfgen computes equiprobable cosine bin boundaries and prints them.

3: The data consists of pairs (energy of secondary particle, probability) for a sequence of incident energies and cosines of the collision angle. This data is in the center-of-mass system. The intent is to provide a correlated, (2-dimensional, over cosine and energy of the secondary) probability density, but that is not how the data is normalized. Instead, the integral of the probability density over the energy of the secondary particle is itself 1 . Consequently, the I_number $=3$ data must be sup-

: plemented by I number $=1$ data giving the angular distributions. The code mcfgen first verifies that the I number $=1$ data is present and consistent. It then computes boundaries for joint equiprobable cosine bins and secondary energy bins, and it prints them.

4: The data consists of pairs (energy of secondary particle, probability) for a sequence of incident energies. This data is in the laboratory system. The code mcfgen computes equiprobable secondary energy bin boundaries and prints them.

7: The data consists of pairs (incident energy, multiplicity) for fission neutrons. These neutrons may be prompt or delayed. The code computes 
the averages over the energy bins of the number of neutrons per fission, weighted by the fission cross section. For each energy group $g$ the output of the code is

$$
\bar{\nu}_{g}=\frac{\int_{g} \bar{\nu}(E) \sigma(E) \phi(E) d E}{\int_{g} \sigma(E) \phi(E) d E}
$$

9: The data consists of pairs (incident energy, multiplicity) for emitted photons. The averages are the same as for I_number $=7$.

10: The data consists of pairs (incident energy, energy of secondary particle). This data may be in the center-of-mass system or the laboratory system, depending on the kinematics type of the reaction. For each energy group $g$ the average is

$$
\tilde{E}_{g}=\frac{\int_{g} \widetilde{E}(E) \sigma(E) \phi(E) d E}{\int_{g} \sigma(E) \phi(E) d E}
$$

11: The data consists of pairs (incident energy, energy deposited to the residual nucleus). This data is not used by mcfgen, but it could be used to compute group averages as is done for $I$ number $=10$.

12: The data consists of pairs (incident energy, $Q$ ), where $Q$ denotes the sum of the energies available from the non-elastic reactions. For each energy group $g$ the average is

$$
Q_{g}=\frac{\int_{g} Q(E) \sigma(E) \phi(E) d E}{\int_{g} \sigma(E) \phi(E) d E}
$$

941: Coherent scattering form factors for incident photons. The coherent scattering form factor $F_{R}(E)$ specifies the importance of Rayleigh scattering. Specifically, for Rayleigh scattering from a target with atomic number $Z$ the cross section is $C(Z)\left(1+\mu^{2}\right)$, where $\mu$ is the cosine of the collision angle and $C(Z)$ is a coefficient depending on the target. Then $[1$, p. 3-23] the angular distribution for coherent scattering for such a target is given by the formula

$$
f(E, \mu)=\left[F_{R}(E)\right]^{2} C(Z)\left(1+\mu^{2}\right) .
$$

The data consists of pairs of grid-based data (incident energy, form factor), and this information is printed in the 'mcf.asc' output file. 
942: Incoherent scattering form factors for incident photons. The incoherent scattering form factor $F_{C}(E)$ specifies the importance of Compton scattering. Specifically, the Klein-Nishina formula for the Compton scattering cross section is

$$
K_{N}(E, \mu)=\frac{\left(1+\mu^{2}\right)(1+\alpha x)+(\alpha x)^{2}}{(1+\alpha x)^{3}}
$$

where $E$ is the incident energy, $\mu$ is the cosine of the collision angle, $\alpha$ is the photon energy in units of electron rest energy, and $x=1-\mu$. The angular distribution for incoherent scattering is given by $[1, \mathrm{p} .3-23]$

$$
f(E, \mu)=F_{C}(E) K_{N}(E, \mu) .
$$

The data consists of pairs of grid-based data (incident energy, form factor), and this information is printed in the 'mcf.asc' output file. Incidentally, the energy of the secondary photon (in units of electron rest energy, $\alpha)$ is given by $\alpha /(1+\alpha x)$. 


\section{Kinematics types}

The reactions are classified into 8 different types, depending on the kinematics. The data in the output files mcf.asc and mcf.bin depends on the kinematics type. For each reaction mcfgen computes the flux-averaged cross section over an energy bin ( $I$ number $=0$ data). Additional data is provided, depending on the kinematics type. The different kinematics types are as follows, along with a description of the additional data provided.

Kinematics type 0: Only cross sections are given.

Kinematics type 1: This kind of data describes 2-body interactions in center-of-mass coordinates. The code mcfgen provides angular distributions (based on data of type I number $=1$ ) and energy deposition to the secondary particle (derived from I number $=10$ data). The angular distributions are equiprobable in terms of the cosine of the collision angle in the center-of-mass system.

If the residual nucleus is light enough to be viewed as a particle (an alpha-particle or lighter), energy deposition to it is also provided.

Kinematics type 2: This is one of two primary kinematics types for reactions with more than two final particles, but it also includes additional special cases. One of the special cases of kinematics type 2 is that of capture of the incident particle by the nucleus, with emission only of photons (denoted by $S=3$ ).

All of the data is in the laboratory system. For each secondary particle the code mcfgen prints the following information. It first gives the equiprobable energy distributions (from I_number $=4$ data) for the secondary particle. It may also provide angular distribution data (derived from Inumber $=1$ data), but the angle and energy distributions are uncorrelated. Furthermore, mcfgen gives the energy deposition to the secondary particles ( $I$ number $=10$ ). If multiple photons or fission neutrons are ejected (I_number $=9$ or 7 data), their average multiplicity per energy bin are also given.

Kinematics type 3: This kinematics type also applies to reactions with multiple secondary particles. It differs from kinematics type 2 in that in 
this case mcfgen gives equiprobable joint distributions in collision angle and secondary energy (obtained from I_number $=3$ and I_number $=1$ data).

For the special case of the reaction identifier $C=5$ the flux-weighted average of the energy available from non-elastic reactions (based on I_number $=12$ data) is also given.

Kinematics type 4: For this kinematics type we start with a 2-body reaction in center-of-mass coordinates, but one of the products is an unstable intermediate nucleus. For the first seocndary particle for this reaction mcfgen produces equiprobable angular bins and the average energy deposition per energy bin.

The decay of the intermediate nucleus is treated as in kinematics type 2, with possible decays of further intermediate nuclei all lumped into a single process.

Kinematics type 5: This is a very special case which is currently used only for the reaction $d\left(n, n^{\prime}\right) n p$. We start with a 2-body reaction in center-ofmass coordinates, with sufficient energy to break up the deuteron. The neutron and proton resulting from the break-up of the deuteron simply go off independently at the velocity of the unstable recoil deuteron.

Part of the data produced by mcfgen is that of kinematics type 1 (the collision angle and the energy carried by the recoil neutron). For the products of the broken deuteron, the energy carried by the proton is given.

Kinematics type 6: In this special case the data consists of of form factors for coherent (Rayleigh) scattering of a photon (I_number $=941$ ).

Kinematics type 7: In this special case the data consists of of form factors for incoherent (Compton) scattering of a photon (I_number $=942$ ).

This information is summarized in the following table. In this table the rows are by kinematics type, and the columns by data type (I_number). A check mark indicates that this type of data is sure to be present for the given kinematics type. In addition, there may be multiplicity data, I_number $=7$ or 9 . 
Data Type (Inumber) and Kinematics Type

\begin{tabular}{|c|c|c|c|c|c|c|c|c|}
\hline \multirow{2}{*}{ kin_type } & \multicolumn{7}{|c|}{ I_number } \\
\hline & 0 & 1 & 3 & 4 & 10 & 12 & 941 & 942 \\
\hline 0 & $\sqrt{ }$ & & & & & & & \\
1 & $\sqrt{ }$ & $\sqrt{ }$ & & & $\sqrt{ }$ & & & \\
2 & $\sqrt{ }$ & $\sqrt{ }$ & & $\sqrt{ }$ & $\sqrt{ }$ & & & \\
3 & $\sqrt{ }$ & $\sqrt{ }$ & $\sqrt{ }$ & & $\sqrt{ }$ & $\sqrt{ }$ & & \\
4 & $\sqrt{ }$ & $\sqrt{ }$ & & $\sqrt{ }$ & $\sqrt{ }$ & & & \\
5 & $\sqrt{ }$ & $\sqrt{ }$ & & $\sqrt{ }$ & $\sqrt{ }$ & & & \\
6 & $\sqrt{ }$ & & & & $\sqrt{ }$ & & $\sqrt{ }$ & \\
7 & $\sqrt{ }$ & & & & $\sqrt{ }$ & & & $\sqrt{ }$ \\
\hline
\end{tabular}

The subroutine models in mcfgen determines the kinematics type, based on the properties of the data given above. The code mcfbin transfers this information to the binary output file mcf . bin. 


\section{Interpolation}

The interpolation code intcode used in the ENDL system specifies the type of interpolation to be used for the data on energy and cross section. Only the values intcode $=0$ and 5 are used. In fact, even when the 'mcf.asc' file specifies log-log interpolation, the data contains sufficiently many points so that linear-linear interpolation is accurate to with $0.1 \%$.

intcode $=0$ : Linear-linear interpolation. This is used for all but incident gammas.

intcode $=1$ : Piecewise constant (a histogram).

intcode $=2$ : Linear-linear interpolation.

intcode $=3$ : Linear-log interpolation (logarithmic in $x$ ).

intcode $=4:$ Log-linear interpolation (logarithmic in $y$ ).

intcode $=5:$ Log-log interpolation. This is used only for incoming gammas. 


\section{File formats}

In this chapter we explain in detail the nature of the files associated with the MCF system. We start with the input files 'mcfgen.input' and 'library' for mcfgen, and we then describe its output file 'mcf.asc'. Finally, we give the format of the binary output 'mcf.bin' of the code mcfbin.

\section{The file 'mcfgen.input'}

The usual purpose of the 'mcfgen.input' file is to specify the incident particle and the range of targets, but it may also be used to tailor the processing of data by mcfgen. In particular, while the default source of much information is the file 'bdfls', we may override this by the insertion of flags into 'mcfgen.input'.

\section{Structure of the file 'mcfgen.input'.}

The following is a typical first line of the file 'mcfgen.input',

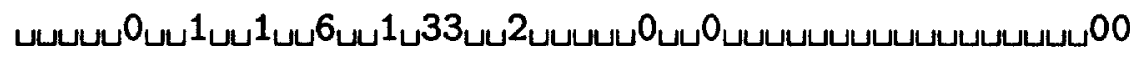

(In practice several of the zeros are usually replaced by spaces, but these spaces are read as zeros.) The spacing is rigidly prescribed by the format,

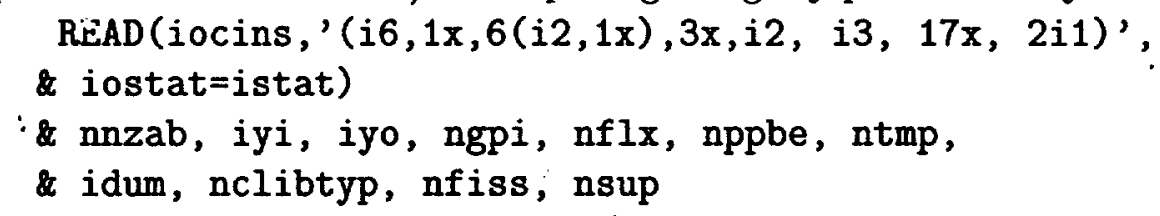

The meanings of these values is as follows.

nnzab: The atomic number and mass number $(1000 * Z+A)$ for this target. The value nnzab $=0$ is most common, and it serves as a flag indicating that nnzab is overwritten by the next line in the 'mcfgen.input' file. A value of $A=0$ denotes the natural element, so that $Z A=28000$ represents natural nickel. The special values $Z A=99120$ and 99125 denote, respectively, short-lived and long-lived fission fragments. 
iyi: The identifier for the incident particle. See the documentation for 'particle' in the chapter on Various identifiers. In the example above the ' 1 ' denotes a neutron.

iyo: The identifier for the outgoing particle. In the example line we have a neutron, iyo $=1$. This parameter is not used.

ngpi: If ngpi $>0$, it identifies which energy group bounds are to be read from the 'bdfls' file. In the instance above we have ngpi $=6$, so we take the collection of energy groups labeled ' 6 ' (175 groups with $20 \mathrm{Mev}$ maximum). Note that for incident charged particles we usually have ngpi $=71$ giving energy 63 groups with $20 \mathrm{Mev}$ maximum, and for incident photons the standard value is $\mathrm{ngpi}=80$ for 175 groups with $30 \mathrm{Mev}$ maximum. In order to get the energy group boundaries from the 'mcfgen.input' file, one sets ngpi $=0$.

nflx: A flux identifier. This is the flag specifying flux data in the 'bdfls' file. The value $n f l x=1$ gets an energy-independent flux. In order to get a non-constant flux, one may specify one of the other flux identifiers in the 'bdf $1 \mathrm{~s}$ ' file, or one may set $\mathrm{nfl} \mathrm{l}=0$, denoting that flux is given in the 'mcfgen.input' file.

nppbe: The number of equiprobable angular bin boundaries (33 here) for data type I_number $=3$. This is reset to 17 if it is 0 here. The number of equiprobable angular bins is one less.

ntmp: The temperature identifier. Usually, ntmp $>0$ and it is the flag for temperature data in the 'bdfls' file. In the example it is ntmp $=2$, giving the set ' 2 ' of temperatures. If ntmp $=0$, we get the temperatures from the 'mcfgen.input' file. In fact, there is to be only one temperature because Doppler broadening is not done within mcfgen.

idum: Unused (an old debug option).

nclibtyp: Defines the data type:

nclibtyp $=0:$ The output is in terms of bin averages.

nclibtyp $=1$ : The output is grid based. This option is used only for incident photons. 
nfiss: Not used by mcfgen.

nsup: The temperature or level range request sentinel.

nsup $=0:$ No special excitation levels or range of temperatures. This is the usual choice.

nsup $=1:$ Read in the range of excitation levels from the input file 'mcfgen .input'.

nsup = 2: Get the range of temperatures from the 'mcfgen.input' file. But note again that there can be only one temperature because mcfgen does not handle Doppler broadening.

nsup $=3$ : Read in the ranges of excitation levels and temperatures from the 'mcfgen.input' file.

\section{Optional additional lines in the 'mcfgen.input' file.}

The first line of 'mcfgen.input' usually has nnzab $<1$, in which case the second line of the file gives the range of targets in terms/ of the values of $1000 * Z+A$. The format is:

READ (iocins, '(2i6)') nnzab, nnzae

A typical line is

பบபบบ1ப99125

In this case the target ranges from a neutron $Z A=1$ to a fission fragment $Z A=99125$. It is very common to use this option because the standard first line of the 'mcfgen.input' file permits the specification of only a single target.

The remaining options are usually not used, and they are given here only for the sake of completeness. These options all depend on the values of flags as described above in the discussion of the first line of the 'mcfgen.input' file.

If nsup $=1$ or nsup $=3$, the next line of the 'mcfgen.input' file gives the range of excitation levels in the format:

READ (iocins,'(2e11.4)') elvb, elve 
If nsup $=2$ or nsup $=3$, the next line of the 'mcfgen.input' file gives the range of temperatures in the format:

$$
\text { READ (iocins, '(2e11.4)') tempb, tempe }
$$

If ngpi $=0$, we get the energy bin boundaries from the 'mcfgen.input' file. But we first read the number of bin boundaries.

READ (iocins, '(i4)') nbounds

READ (iocins, ' $(6 \mathrm{e} 12.5)$ ') (bounds $(i), i=1$, nbounds)

Ordinarily, this information is read from the 'bdfls' file.

If $n f l x=0$, we get the flux from the 'mcfgen.input' file. We first read the order of the Legendre expansion Imax, which must be zero. We then read nflux, the number of (energy, flux) entries. Finally, we read the (energy, flux) pairs.

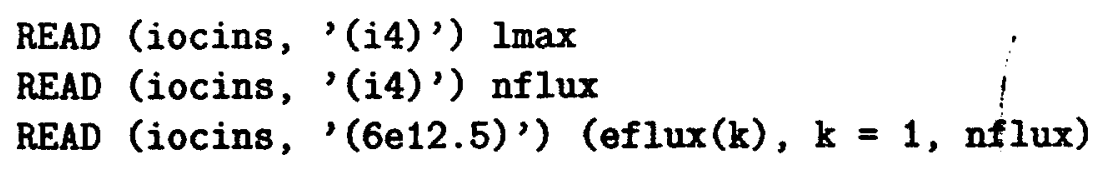

If $n t m p=0$, we get the temperatures from the 'mcfgen.input' file.

$$
\begin{aligned}
& \text { READ (iocins, ' }(i 4,68 x, i 1) \text { ') numtemp } \\
& \text { Read (iocins, ' } \left.(6 e 12.5)^{\prime}\right) \text { (temture }(i), i=1 \text {, numtemp) }
\end{aligned}
$$

Otherwise, mcfgen reads this information from the 'bdfls' file. Note again that numtemp must be 1 because Doppler broadening is not implemented here. 


\section{The format of the 'library' file}

The 'library' file contains the assembled evaluated data for all of the targets. This is a binary file, and it is written under the assumption that integers and real numbers occupy the same amount of storage. This is the principal portability issue for mcfgen. The 'library' file starts with an overall directory containing identification, a list of targets, and a list of offsets to the information on each target. The block for each target starts with a target directory giving basic information about the target, a list of reactions and the types of data given for each reaction, as well as the location of this data in the file.

\section{Background information}

The library files endl for incident neutrons, ecpl for incident charged particles, and egdl for incident photons are documented in the references [3] and [5]. These libraries are derived from ASCII data files located in subdirectories under

$$
\text { /nds/data/endl, /nds/data/ecpl, /nds/data/egdl }
$$

on the Nuclear Data Group's network. These ASCII files are arranged in separate directories for the different targets, and there is a separate file for each property of each reaction, with the identification incorporated in the name of the file. Thus, the file

$$
\text { /nds/data/endl/za016032/yo07c55i001s003 }
$$

pertains to incident neutrons on the target ${ }^{32} \mathrm{O}$, the ejected particle is a photon (yo $=7$ ), the reaction is photon production $(\mathrm{C}=55)$, and the data gives angular distributions ( $I$ number $=1$ ). Furthermore, the value $S=3$ indicates that the energy of the emitted photon is given by $X_{1}$.

In order to create a library, see the script

$$
\text { /nds/bin/build.mcf.general }
$$

There are two steps involved. The first step is to run endlret, to make an ASCII file containing all of the data for the particular incident particles reacting with all of the targets. The second step is to run create, to convert the ASCII file to binary. 


\section{The overall directory}

The directory information is as follows, with the names of the variables as in the subroutine libhdr, where this data is read from the 'Iibrary' file.

libid: This is intended to be identification for the library in a packed format, but it is not clear what the packing is, since all of our libraries have the same identifier. The reference [3] gives an explanation, but it is clearly not followed.

libdate: The date the library was created. Thus, 930914 represents 14 September 1993.

isonum: The number of target isotopes in the input file.

zas: An integer array of the $\mathrm{ZA}$ numbers $1000 * Z+A$, atomic number and mass number for targets in the input file. This array is declared in the file 'iodata.h', where it is called isolst. A value of $A=0$ denotes the natural element, so that $Z A=28000$ is natural nickel. Also, the special values $Z A=99120$ and 99125 denote, respectively, short-lived and long-lived fission fragments.

levels: The excitation levels for the targets.

abunds: Abundances are read in but never used.

temp: The temperatures of the targets.

tdadds: An integer array of addresses of the individual target directories. 


\section{The target directory}

The block of information for a target in the 'library' file starts with a target directory, and this is followed by the actual data. The target directory is read from the 'library' file by the subroutine trghdr, and it is stored under the equivalenced arrays nhead and xhead. Each item in the target directory (REAL or INTEGER) is 64 bits long, and this fact makes for portability problems. The form of the target header is as follows. The information presented here is derived from the note [5] by Rathkopf.

\section{Identification of the target.}

We start with the quantities:

nnza: The atomic number and mass number for the target. The format is $1000 * Z+A$, but there are special cases. The value $A=0$ denotes a natural element, so that nnza $=8000$ for natural oxygen. Also, the values nnza $=99120$ and 99125 are used for fission fragments. Furthermore, in some instances nnza $=-9$ denotes an electron.

atm: The atomic mass of the target.

elevel: The excitation level of the target.

halflife: The halflife. Stable isotopes are given a halflife of $10^{50}$.

(date, nwd): Two 32-bit integers, containing the date of the most recent update for this target and the length of the target directory:

ndàtsyz: The number of data words following the directory.

numc: The number of reactions for the target.

\section{Locations of the reaction information.}

There now comes numc pairs of numbers.

C: The reaction identifier. See the section on ' $\mathrm{C}$ reaction identifiers' in the chapter on Various identifiers.

C fwa: Where in nhead to find the offsets to the data for this reaction. 


\section{Data identification and location.}

At this point we have numc blocks telling the types of data given for each reaction and where in nhead to find the offsets for this data.

numi: The number of data blocks for this reaction. We then have numi sets of the following 4 numbers.

rpid: A packed word containing the data identifier I_number, the date, the identity of the first secondary particle, and the interpolation flag.

Ifwa: The location in nhead of the offsets for the beginning and end of this block of data.

emin: The minimum energy for this reaction.

emax: The maximum energy for this reaction.

\section{Offsets of the data blocks.}

There now follows large blocks telling where the data is located, one block for each of the numc reactions. Note: each of the following locations is relative to the start of the data for this target. Thus, the first data location is 1 .

C_data_fwa: The location of the start of the data for this reaction.

C_data_lwa: The location of the end of the data for this reaction.

yi: The identification of the incident particle.

date: The date of the latest update for this reaction.

S: The S reaction switch. See the section on it in the chapter on Various identifiers.

Q0: The energy of the reaction.

$\mathrm{X1}, \mathrm{X} 2, \mathrm{X3}, \mathrm{X} 4$ : Additional data, depending on the value of $\mathrm{S}$.

Data locations: We now have the information for each of the data blocks for this reaction. The content of these sets is explained below. 


\section{Locations of the data blocks.}

For each reaction there several blocks of data. The section of nhead devoted to data location and identification provides the number numi of these blocks, the data identification (Innumber), and the locations of the data-location blocks. The data-location portion of the target directory contains additional information, depending on the value of I number as follows. Again the data locations here are in terms of the start of the data for this target.

I_number $=0:$ For the reaction cross sections we have two offsets.

data_fwa: The location of the start of the cross section data for this reaction.

data_lwa: The location of the end of the cross section data.

I_number $=1:$ For the angular distributions the data consists of pairs (cosine, probability density) for a number of incident energies. The corresponding set in nhead contains the following information.

data fwa: The location of the start of the angular distribution data for this reaction. This is the location of the incident energies.

data_lwa: The location of the end of the angular distribution data.

max_cos_prob: The maximum number of words of angular distribution data for each incident energy.

num_engy_in: The number of incident energies.

: I1_data_fwa: Here are num_engy_in numbers: the location of the start of the angular distribution data for each incident energy.

I_number $=3$ : This block of data corresponds to correlated probability of angle and energy of the secondary particle. The data block starts with the incident energies. For each incident energy we then have the cosines, followed by the corresponding pairs (energy of secondary particle, probability density). This set in nhead contains the following information.

data fwa: The location of the start of the correlated distribution data for this reaction. This is the location of the incident energies. 
data_lwa: The location of the end of the correlated distribution data. num_engy_in: The number of incident energies.

n3cosmax: The maximum number of cosines for each incident energy. nE_out_max: The maximum amount of (E', probability density) data for each incident energy and each cosine. There now follows num_engy_in blocks.

I3_ptr: The location of the cosines for this incident energy.

n3cos: The number of cosines for this incident energy.

e_prob_ptr: We have here n3cos locations of the starts of the energy distribution data for this incident energy and cosine.

Innumber $=4:$ For the energy distributions the data consists of pairs (energy of secondary particle, probability density) for a number of incident energies. The corresponding set in nhead contains the following information.

data_fwa: The location of the start of the energy distribution data for this reaction.

data_lwa: The location of the end of the energy distribution data.

lmax: The order of the Legendre expansion of the flux. This must be zero.

max_num_engy_in: Unused by mcfgen.

lep: The maximum number of words of energy distribution data for each incident energy.

energy_fwa: The location of the incident energies.

num_engy_in: The number of incident energies.

I4_data_fwa: A collection of num_engy_in locations of the starts of the energy distribution data for this incident energy.

I_number $=7:$ The data is the number of neutrons per fission, prompt or delayed, and we have two offsets. 
data_fwa: The location of the start of the neutron multiplicities.

data_lwa: The location of the end of the neutron multiplicity data.

I number $=9:$ The data is the number of photons emitted, and we have two offsets. We have two offsets.

data fwa: The location of the start of the multiplicity data for this reaction.

data_lwa: The location of the end of the multiplicity data.

I number $=10$ : For the energy depositions to secondary particles we have two offsets.

data fwa: The location of the start of the energy deposition data for this reaction.

data_lwa: The location of the end of the energy deposition data.

Innumber $=11:$ For the energy depositions to the residual nucleus we have two offsets. This data is currently not used.

data_fwa: The location of the start of the energy deposition data for this reaction.

data_lwa: The location of the end of the energy deposition data.

I number $=12:$ For the energy production by the non-elastic reactions we : have two offsets. This data is used only for production cross sections $(\mathrm{C}=5)$.

data_fwa: The location of the start of the energy deposition data for this reaction.

data_lwa: The location of the end of the energy deposition data.

I_number $=941:$ For the coherent scattering form factors we have two offsets.

data.fwa: The location of the start of the form factor data for this reaction. 
data_lwa: The location of the end of the form factor data.

I_number $=942:$ For the incoherent scattering form factors we have two offsets.

data_fwa: The location of the start of the form factor data for this reaction.

data_lwa: The location of the end of the form factor data. 


\section{The format for 'mcf.asc'}

The purpose of the code mcfgen is to produce an ASCII file 'mcf.asc'. The information in this file is used by the code mcfbin to produce a binary file 'mcf.bin' for use in all-particle Monte Carlo codes. The 'mcf.asc' file begins with two introductory lines, followed by a loop over the targets. For each target the first few lines provide its identity and some basic information, including a list of the reactions. The first target is special, in that its data starts with the energy bin boundaries and the temperature.

For each of the targets we have a loop over the reactions, and for each reaction the file contains data for various reaction properties, such as cross section, angular distribution of ejecta, and energy distribution. Each of these data blocks is preceded by an identification flag and a header, and this flag and header information are essential to the mcfbin code. The end of the data for a target is marked by a flag (the number 99).

The notification for the end of the file is a particular phoney target $Z A=$ 999999.

In summary, the form of an 'mcf .asc' file is as follows,

First line: The date this 'mcf.asc' file was created, and its type.

Second line: Identify the library source and the date of the latest modification of the library.

Loop over the target nuclei.

Identify the target: A new target is marked by ' $*$ ' at the end of the line. The rest of the line contains this target's atomic number and mass number, the number of incident energy groups, and the number of reactions.

Basic target data: Identify the incident particle, the halflife, the excitation level of the target, the temperature of the target, the target's atomic mass, and the library date.

List the reactions: Next in the file follows the list of values of the $\mathrm{C}$ reaction identifier for this target.

For the first target: If this is the first target in the 'mcf.asc' file, then come the energy bins and the target temperature as follows. 
Energy bin block: One line for the energy-bin flag and the number of bins. Then come the energy bin boundaries.

Temperature block: This option exists in case we someday implement Doppler broadening inside mcfgen. For now, there is just one target temperature. The file has one line for the temperature flag and the number of temperatures, and it has another line for the temperature.

Loop over the reactions: We go tirrough the reaction identifiers (Cvalues) for this target.

Loop over the data for a reaction: For each reaction (i.e., for each C-value) for this target there are various blocks of data, according to I_number. Each block is of the following form. The first line of the block starts with the flag for the type of data, and it may have other information. The second line is a header giving the amount of data and further identification of the data. The data then follows.

Flag 99: A special flag marks the end of data for a target.

End of file: The phoney target $1000 * Z+A=999999$ marks the end of the file.

\section{Specifics.}

For the reader who needs to know the exact format and content of the 'mcf.asc' file, it is as follows.

First line: The form of the first line is,

WRITE (iocmcf,

\& '("MCFGEN Date:", i8, 5x, "Library type:", i3)')

\& i_date, nclibtyp

Here, the date is in the format such that 12 January 1995 is written as 950112. The code for the library type is 
nclibtyp $=0$ means bin-average data.

nclibtyp $=1$ means grid-based data.

Second line: The library source and the date are given as

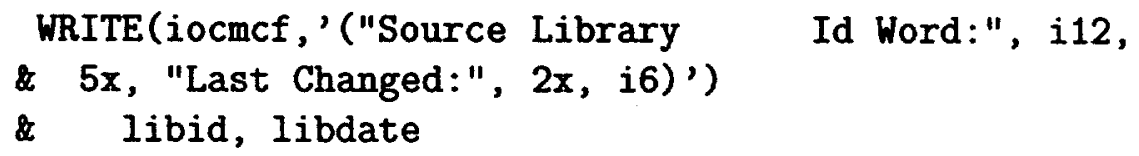

Here, libid is meant to be the library identifier packed in the format, but it is not clear what the components are. The convention given on page 16 of reference [3] is not followed because all of our 'mcf.asc' files have libid $=103940000$.

Identify the target: A line identifying this target, the number of energy groups, and the number of reactions.

WRITE (iocmcf,'(i6, 12x, i3, 3x, i3, 44x,"*")')

\& nnza, negi, numcs

Here, the meaning of the entries is:

nnza: The atomic number and mass number of the target, formatted as $1000 * Z+A$. There are some special conventions. A value of $A=0$ denotes the natural element, so that nnza $=8000$ is natural oxygen. The value nnza $=99120$ denotes a short-lived fission fragment, and the value nnza $=999999$ is a flag marking the end of the file.

negi: The number of energy groups.

numcs: The number of reactions (C-values) for this target.

*: The final "*" on the line is a flag showing that we are starting data for a new target. 
Basic target data: Identify the incident particle, the halflife, the excitation level of the target, the temperature of the target, the target's atomic mass, and the library date.

$$
\begin{aligned}
& \text { WRITE(iocmcf,'(1p, i2,4e11.4,1x,i6)') } \\
& \text { \& iyi, halflife, elvfd, tmpfd, atm, rpdate }
\end{aligned}
$$

The meaning of the entries is:

iyi: The identifier for the incoming particle.

halflife: The halflife of the target. A stable target is given a halflife of $10^{50}$.

elvfd: The excitation level of the target.

tmpfd: The temperature of the target.

atm: The atomic weight of the target.

rpdate: The date of the data for this target.

List the reactions: Then follows the list of values of the $\mathrm{C}$ reaction identifier for this target.

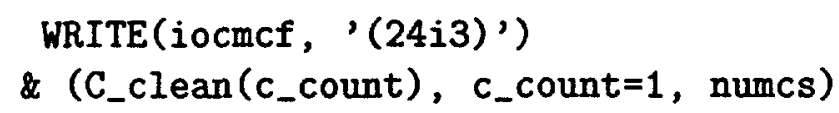

Data block: A data block takes the following form.

Flag: A single line, starting with a flag to identify the type of data. The most common format for the data-flag line is,

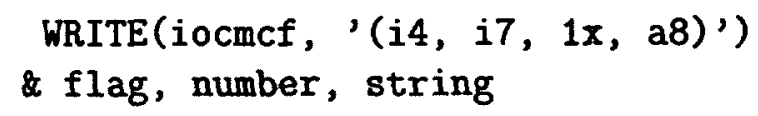

Header: There is usually a second line identifying the reaction and the type and number of data entries. 
Data: The actual data.

The type of data is identified by means of the flag. The dentification is unique in all cases except that $\mathrm{flag}=12$ has two interpretations-its most common usage is as the flag for energy distributions ( $I$ _number = 4), but it also used for the angular distribution of gammas. The header line must be used to distinguish them. A point to be aware of is that for $f l a g=1$ and 2 there is no header line. But these two data blocks appear only with the first target, and they come immediately after the list of reactions, before the rest of the data. We list the types of data blocks ordered in terms of flag because that is what is used by the code mcfbin which reads the 'mcf.asc' file. The user who is accustomed to working in terms of the data identifier I number should consult the following list.

I_number $=0:$ Cross sections, data with $\mathrm{flag}=4,5$, and 6 are given, in that order.

I_number $=1:$ Angular distributions, $f l a g=10$ and sometimes 12 . When $f l a g=12$ data is present, it comes first.

I_number $=3$ : Correlated energy-angle distributions, $f l a g=11$.

I_number $=4:$ Energy distributions, $f l a g=12$.

I_number $=7:$ Fission neutron multiplicities, $f l a g=3$.

Inumber $=9:$ Photon multiplicities, $f l a g=9$.

I_number $=10:$ Energy deposition, $f \operatorname{lag}=7$.

: $\quad$ Inumber $=12:$ Average available energy, $f l a g=6$.

$I$ number $=941$ : Coherent scattering form factors, $f l a g=14$.

I number $=942:$ Incoherent scattering form factors, flag $=15$.

The content of the data blocks as ordered by flag is as follows.

flag $=1$ : This block of data is energy bin boundaries, number is the number of bins, and string is "gp bds". There is no header, and the energy bin boundaries follow immediately, written with the format, 
WRITE (iocmcf, '(1p, 6e11.4)') (ebound(j), j=1, nebi)

flag = 2: This block of data is target temperatures, number is the number of temperatures, and string is "temp.". There must be only one temperature because Doppler broadening is not now done in mcfgen. That temperature is given on the next line in the format,

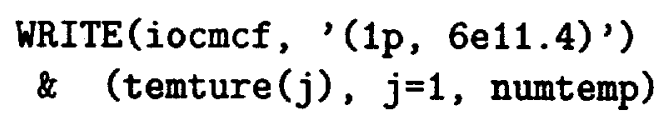

flag = 3: This block of data is multiplicity of fission neutrons. The format of the flag line is nonstandard,

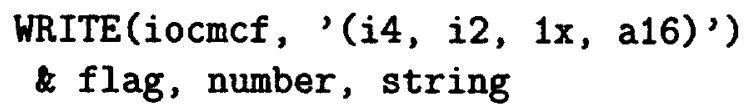

For prompt neutrons we have number $=0$ and the label string is "nubar, prompt". For delayed neutrons we have the identifier number = 1 and string is "nubar, delayed". The flag line is followed by a header of the form,

$$
\begin{aligned}
& \text { WRITE(iocmcf, '(1p, } \left.5 i 5,5 x, 3 e 12.5)^{\prime}\right) \\
& \text { \& } \text { C, I_number, s, igsrt, igend, } q 0, x 1
\end{aligned}
$$

The meaning of these entries is:

c: The $\mathrm{C}$ reaction identifier ( $\mathrm{C}=15$ for fission).

I_number: For $f l a g=3$ the data identifier I_number is 7 .

s: The $\mathrm{S}$ reaction identifier. It determines the meaning of $x 1$.

igsrt: Identify the first nonzero cross section. (This amounts to an energy threshold.)

igend: Identify the highest nonzero cross section. (In effect, this is a maximum energy.)

q0: The energy of the reaction.

$x 1$ : The first extra information, depending on the value of $s$. 
The group-averaged multiplicities are printed in the format,

$$
\begin{aligned}
& \text { WRITE(iocmcf, '(1p, 6e11.4)') } \\
& \&(\operatorname{array}(j), j=i g s r t, \text { igend) }
\end{aligned}
$$

flag $=4:$ This block of data is flux-weighted group-averaged cross sections, number is the date for the data, and string is "gp $x$-sec". The flag line is followed by a header of the form,

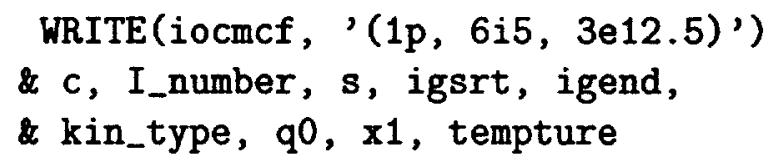

The differences from the header for $f l a g=3$ are I_number: The data identifier is 0 .

kin_type: The kinematics type for this reaction. tempture: The temperature of the target.

The cross section data follows,

$$
\frac{\int_{g} \sigma \phi d E}{\int_{g} \phi d E}
$$

and it is written in the following format. Note that the zeros below the threshold and above the maximum are not printed.

$$
\begin{aligned}
& \text { WRITE(iocmcf, '(1p, 6e11.4)') } \\
& \text { \& (gpsig0 }(j), j=\text { igsrt, igend) }
\end{aligned}
$$

The cross section data is followed immediately in the file (without an identification flag) by a line containing the number of particles produced by this reaction, including the residual nucleus.

$$
\text { WRITE (iocmcf, '(i2)') parts_produced (1, nrc) }
$$

Then come the secondary particles and their multiplicities as pairs (yo, multiplicity). The residual nucleus is the last particle, unless it is light enough to be included with the other secondary particles. 
WRITE(iocmcf, '(15i6)') (parts_produced (j, nrc), \& $\mathrm{j}=2$, parts_produced $(1, \mathrm{nrc}) * 2+1)$

flag $=5:$ This block of data is the calculated energy available, and it always follows the block for $f l a g=4$. The flag line is nonstandard in that number is not present. The string in the flag line is "en avail". The header line from $f l a g=4$ is printed again. (We again have I_number $=0$.) The available energies per group

$$
\frac{\int_{g} E \sigma \phi d E}{\int_{g} \sigma \phi d E}
$$

are then given with the format,

$$
\begin{aligned}
& \text { WRITE(iocmcf, '(1p, 6e11.4)') } \\
& \text { \& (av_en_avail }(j), j=i g s r t, ~ i g e n d)
\end{aligned}
$$

$\mathrm{flag}=6$ : If this block of data is present, it appears immediately after the block for $f l a g=5$. It contains the energy available as given in the Production Cross Section library file. In the flag line number is not present, and string is "Q-bar". The header line from $\mathrm{flag}=4$ is printed again. (We again have I_number $=0$, but now $C=5$.) The available energies per group are then given with the format,

$$
\begin{aligned}
& \text { WRITE(iocmcf, '(1p, 6e11.4)') } \\
& \text { \& (qbargp }(j), j=i g s r t, \text { igend) }
\end{aligned}
$$

$\mathrm{flag}=7:$ This block of data is energy carried by a secondary particle, number identifies the first of the list of secondary particles, and string is "edeptoyo". The flag line is followed by the same header as for flag $=3$. The only difference is that the data identifier $I$ number is now 10 . The data is

$$
\frac{\int_{g} \tilde{E} \sigma \phi d E}{\int_{g} \sigma \phi d E}
$$

and is given in the format, 


$$
\begin{aligned}
& \text { WRITE(iocmcf, '(1p, 6e11.4)') } \\
& \text { \& (e10barg(io), io = igsrt, igend) }
\end{aligned}
$$

flag $=9:$ This block of data is group-averaged photon multiplicity, number identifies the first of the list of secondary particles, and we have "multipl." for string. The flag line is followed by the same header as for $f l a g=3$. The only difference is that the data identifier I_number is now 9 . The data

$$
\frac{\int_{g} \bar{\nu} \sigma \phi d E}{\int_{g} \sigma \phi d E}
$$

is in the format,

$$
\begin{aligned}
& \text { WRITE(iocmcf, '(1p, 6e11.4)') } \\
& \text { \& (array }(j), j=\text { igsrt, igend) }
\end{aligned}
$$

flag $=10:$ This block of data gives equiprobable angular bins. The number identifies the first of the list of secondary particles, and we have "ang $(i=1)$ " for string. The flag line is followed by the header,

$$
\begin{aligned}
& \text { WRITE(iocmcf, '( } 1 p, 4 i 5,5 x \text {, i5, } 3 e 12.5)^{\prime} \text { ) } \\
& \text { \& c, I_number, s, num_engy_in, neqpb, q0, } x 1
\end{aligned}
$$

For this value of the flag the data identifier I_number is 1 . The unusual item in the header is neqpb, the number of equiprobable angular bin edges. (The number of bins is one less.) The first data entries are the incident energies in the format,

$$
\begin{aligned}
& \text { WRITE(iocmcf, '(1p, 6e11.4)') } \\
& \text { \& (energy_in(k), } k=1, \text { num_engy_in) }
\end{aligned}
$$

The equiprobable angular bin boundaries are concatenated into a single long array, with a block for each incident energy. The entries are printed in the format, 


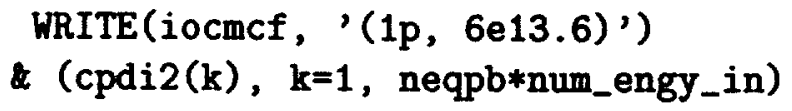

flag $=11:$ This block of data gives correlated bins which are equiprobable jointly with respect to angle and energy. The number in the flag line identifies the first of the list of secondary particles, and string is "ang $(i=3)$ ". The flag line is followed by the header,

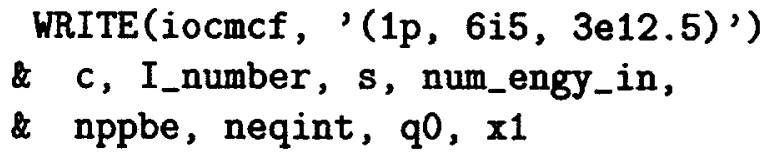

Here, the value of I_number is 3 . The number of equiprobable angular bin edges is nppbe, and the number of equiprobable energy bin edges is neqint. Just as for $\mathrm{flag}=10$ (equiprobable energy distributions), the block starts with the incident energies in the format,

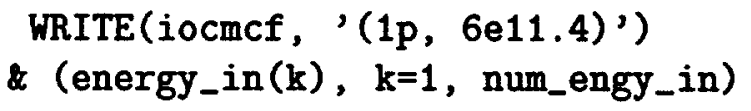

The joint energy-angle distributions are printed in sections. Each section contains, first, an equiprobable angular bin boundary point

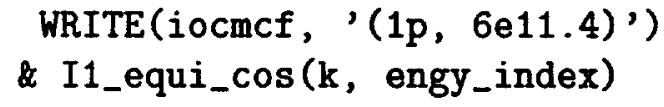

There follows the equiprobable energy bin boundaries,

$$
\begin{aligned}
& \text { WRITE(iocmcf, '(1p, 6e13.6)') } \\
& \& \text { (equi_engy }(j), j=1, \text { neqint) }
\end{aligned}
$$

flag $=12$ : This flag has two possible meanings, and they ought to be split. In both uses number identifies the first of the list of secondary particles, and string is "e spec". The two meanings of the flag are distinguished by the different header lines: 
Energy distributions: For Inumber $=4$ the flag 12 denotes equiprobable energy distributions. The header is,

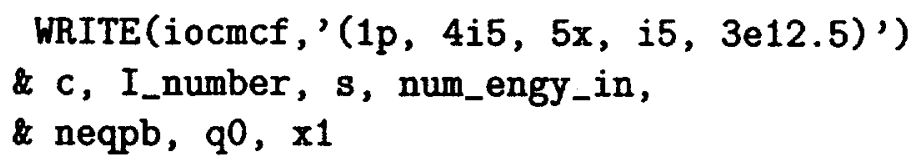

This is standard header information except for neqpb, the number of equiprobable energy bin edges. (The number of bins is neqpb - 1.) The data is in two sets. First, we have the incident energies,

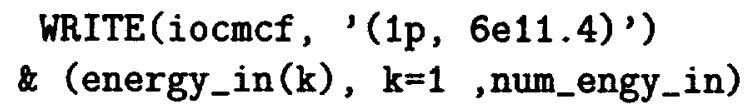

The equidistributed energy deposition bin boundaries for the various incident energies are concatenated into a single array. This array is printed in the format,

$$
\begin{aligned}
& \text { WRITE(iocmcf, '(1p, 6e13.6)') } \\
& \text { \& (cpdi2(k), } k=1, \text { neqpb*num_engy_in) }
\end{aligned}
$$

Gamma production: If I_number $=1$ and $S=3$ (so that the data gives angular distribution for gamma production), this block appears before the angular distribution data (which has $\mathrm{flag}=10)$. There is a special header,

$$
\begin{aligned}
& \text { WRITE(iocmcf, '(1p, } \left.5 i 5,5 x, 3 e 12.5)^{\prime}\right) \text {. } \\
& \& \text { c, I_number, } s, 2,2, q 0, x 1
\end{aligned}
$$

In this case $x 1$ is the energy of the ejected gamma. This line is followed by the energy range

WRITE(iocmcf, '(1p, 6e11.4)') emin, emax

and by the energy of the ejected gamma, four more times!

$$
\text { WRITE(iocmcf, '(1p, } \left.4 \mathrm{e} 13.6)^{\prime}\right)(\mathrm{x} 1, \mathrm{i} 0=1,4)
$$

Note that this is the only situation in which the values of emin and emax get printed, even though they are present with all 
of the data in the 'library' file.

flag $=14$ : This block of data gives coherent scattering form factors. In the flag line number identifies the first of the list of secondary particles, and string is "coh.scat". The flag line is followed by the header,

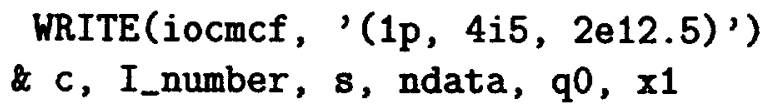

Here, the value of I_number is 941 , and ndata is the length of the data. The scattering form factors are given in the format,

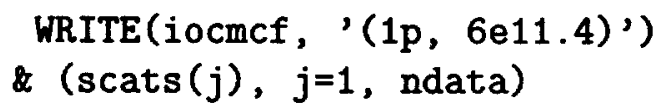

flag $=15:$ This block of data gives incoherent scattering form factors. In the flag line number identifies the first of the list of secondary particles, and string is "inc.scat". The header and the data block are the same as for $f l a g=14$. The only difference is that I_number is 942 .

flag $=99:$ There is no more data for this target.

Incidentally, if nclibtyp $=1$ (grid-based data), there is an extra phoneytarget line containing the interpolation type used for each value of I_number. The format of this line is,

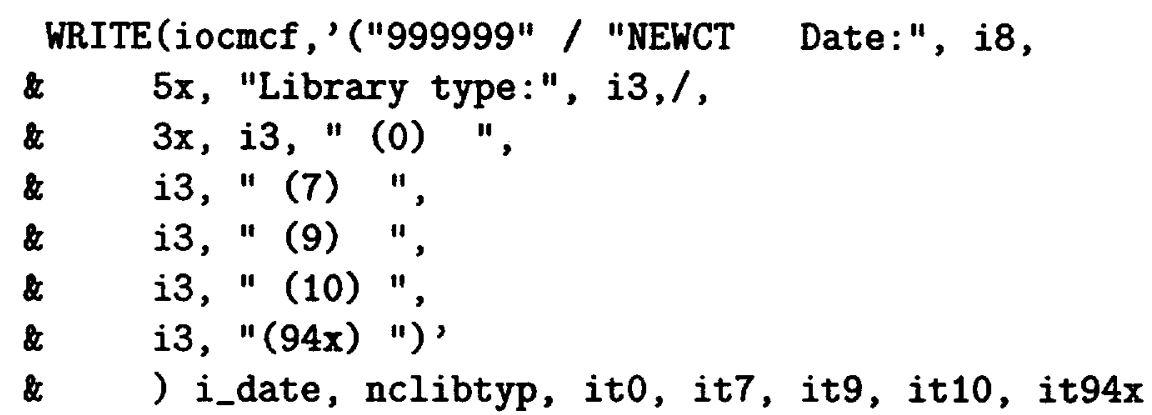

These entries have the following meanings. 
i_date: The date the output file was made.

nclibtyp $=1$ Indicates grid-based data.

itI, "(I)": For each value of I_number what sort of interpolation to use. In any case, there are enough data points, so that linear-linear interpolation is accurate to with $0.1 \%$. The only values of it I used are:

itI $=0$ : Linear-linear interpolation.

itI $=5:$ Log-log interpolation. 


\section{The format for 'mcf.bin'}

The information content of an 'mcf .bin' file is the same as the corresponding ' $m c f . a s c$ ' file. There is some reordering of the data, but the principal difference is that 'mcf.bin' files are in binary format. We acknowledge that the material in this section is taken directly from Perkins's draft report [4].

In general terms, the layout of an 'mcf.bin' file is as follows. The data is organized by reaction for each target, and to identify and locate the data the file contains four types of structures,

Overall directory: The 'mcf.bin' file starts with this structure, which identifies the targets and tells where in the file to find each target directory.

Target directory: The block of the file pertaining to a target starts with its target directory, which gives the number of reactions and tells where to find each reaction directory.

Reaction directory: The reaction directories for a target follow immediately after the target directory. A reaction directory identifies the reaction and gives the energy produced. It also identifies the kinematics type and tells the location of the corresponding kinematics directory.

Kinematics directory: The kinematics directories for a target are located in the 'mcf .bin' file right after its last reaction directory. A kinematics directory identifies the secordary particle and gives the length and location of each block of data. The kinematics data for a target starts

: immediately after its final kinematics directory.

Hence, if we want to locate the energy-distribution data for a certain secondary particle for a certain reaction and a certain target, we first look in the overall directory to get the location of the target directory. We then look in the target directory to get the location of the desired reaction directory. From the reaction directory we find the location of the kinematics directory, which in turn tells us the length and location of the data for the reaction, including that for the energy distribution.

With this general picture in mind, let us proceed to the fine details. We take a bottom-up approach, starting with the kinematics directories. 


\section{The segments of a kinematics directory.}

The kinematics directories differ considerably in form. This is because different kinds of data may be given, depending on the kinematics type of the reaction. Any kinematics directory is, however, built up of some number of the following segments. Note that all data-location entries are relative to the start of the data block for the target. This reference location is given in the target directory.

Identification of secondary particle: The following four numbers.

yo: The particle-number identifier.

ZA: The secondary particle identified as $1000 * Z+A$.

Excitation: The excitation level of the secondary particle.

Mass: The atomic mass of the particle.

Scatterer identification: This block of three numbers is given for reactions involving the scattering of photons.

ZA: The convention is that an electron has $Z A=-9$.

yo: For an electron we have yo $=9$.

Mass: The atomic mass of the scatterer.

Angular bins: For I_number $=1$ data we have four numbers.

: Number of bin edges: The number of the equiprobable angular bin boundaries.

Number of energies: The number of incident energies for which data is given.

Bin location: The location of the first angular bin for the first (smallest) incident energy. For each incident energy the bin edges are ordered by increasing cosine.

Energy location: The location of the list of incident energies. The energies are in increasing order. 
Energy-angle bins: For I_number $=3$ and 1 data we have the following set of numbers.

Number of energies: The number of incident energies for which data is given.

Energy location: The location of the list of incident energies.

Bin locations: We have a block of numbers, one for each incident energy. The data is organized as: the edge of an equiprobable cosine bin followed by the corresponding equiprobable energy bins for the secondary particle. For each incident energy the number given in this segment of the kinematics directory is the location of the first equiprobable cosine bin edge. The cosines are in increasing order, as are the energy bin edges.

Number of cosines: The number of equiprobable angular bin boundaries.

Number of energies for secondary: The number of equiprobable secondary energy bin boundaries.

Block length: The length of a data block corresponding to the bin locations above. This number is equal to the product,

(number of cosines) $*(1+$ number of secondary energies).

Energy bins: For Inumber $=4$ data we have four numbers.

Number of bin edges: The number of equiprobable secondary energy bin boundaries.

Number of energies: The number of incident energies for which data is given.

Bin location: The location of the first secondary energy bin for the first (smallest) incident energy. For each incident energy the secondary energy bins are in increasing order.

Energy location: The location of the list of incident energies. The incident energies are in increasing order.

Multiplicity: For I_number $=7$ and I_number $=9$ data we have either one or five numbers. 
Multiplicity: If the multiplicity of the secondary particle is independent of the energy of the incident particle, it is given here as a real number, and this segment contains no other numbers. A zero here indicates that the multiplicity is energy dependent, and the following four numbers are also present.

Data length: The number of multiplicity-data entries. There is one entry for each energy group.

Data location: The location of the multiplicity data.

gp_start: The index of the first energy group for which data is given. This corresponds to the energy threshold.

gp_end: The index of the last energy group for which data is given.

Energy deposition: For Inumber $=10$ data we the following four numbers.

Data length: The number of energy-deposition entries. There is one entry for each energy group.

Data location: The location of the energy-deposition data.

gp_start: The index of the first energy group for which data is given. This corresponds to the energy threshold.

gp_end: The index of the last energy group for which data is given.

Photon scattering: For I number $=941$ or I number $=942$ data we the following three numbers.

Interpolation type: This number is 5 to denote that log-log interpolation is recommended. Note, however, that linear-linear interpolation will be accurate to within $0.1 \%$.

Data length: The number of scattering form-factor entries. The data is in pairs (incident energy, form factor), ordered by increasing energy.

Data location: The location of the form-factor data. 


\section{The kinematics directories}

We give the structure of the kinematics directories by kinematics type, as defined in the section on 'kinematics type' in the chapter on Various identifiers.

\section{Kinematics type 0.}

For this type there is no kinematics directory because there is no kinematics data.

\section{Kinematics type 1.}

This kinematics type refers to 2-body interactions, and the data is given in terms of center-of-mass coordinates. The kinematics data consists of a collection of equiprobable bins for the cosine of the collision angle (I_number $=1$ data) and a table of average energy depositions to the secondary particle (I_number $=10$ data). In terms of the segments defined above, the contents of a type 1 kinematics directory are as follows.

Directory length: The length of this kinematics directory.

Identification of secondary particle: Identify the secondary particle by yo number.

Angular bins: Location of the I_number $=1$ data.

Energy deposition: Location of the Inumber $=10$ data.

Reserved locations: Two slots reserved for future use.

Residual identification: If the residual is an alpha-particle or lighter, its identity, ZA number, excitation level, and atomic mass are given here. Otherwise, this segment contains four zeros.

Energy deposition: An energy-deposition segment for the residual is given here if the residual is an alpha or lighter. Otherwise, we have four zeros.

Reserved: Two more slots reserved for future use. 


\section{Kinematics type 2.}

This kinematics type usually refers to reactions with more than two final particles, and all of the data is in the laboratory system. Energy distributions ( $I$ number $=4$ data) and energy depositions ( $I$ number $=10$ data) are given for all particles. Uncorrelated angular distributions ( $I$ number $=1$ data) and multiplicities ( $I$ number $=7$ or 9 data) may also be given. Specifically, a type 2 kinematics directory takes the following form.

Directory length: The length of this kinematics directory.

For each particle: A block with the following segments. (The number of particles is given in the reaction directory.)

Identification of secondary particle: Identify the secondary particle by yo number.

Energy bins: Location of the I_number $=4$ data.

Angular bins: Location of the Inumber $=1$ data if it is present in the file. Otherwise, these four numbers are zero.

Energy deposition: Location of the I_number $=10$ data.

Multiplicity: There are three multiplicity blocks, one for photons ( $I$ number $=9$ ), one for prompt fission neutrons (I_number $=7$ and $S=0$ ) and one for delayed fission neutrons (I_number $=7$ and $S=7$ ).

Reserved: Two slots per particle are reserved for future use.

\section{Kinematics type 3.}

This kinematics type also refers to reactions in which several particles are produced, and the data is given in terms of laboratory coordinates. The kinematics data consists of a collection of equiprobable bins for the cosine of the collision angle ( $\mathrm{I}$ number $=1$ data) and correlated energy-angle equiprobable bins ( $I$ number $=3$ data). Energy depositions to the secondary particle ( $I \_$number $=10$ data) and multiplicity ( $I$ number $=9$ data) are also given. The details of a type 3 kinematics directory are as follows.

Directory length: The length of this kinematics directory. 
For each particle: A block with the following segments. (The number of particles is given in the reaction directory.)

Identification of secondary particle: Identify the secondary particle by yo number.

Energy-angle bins: Location of the I_number $=3$ and 1 data.

Energy deposition: Location of the I_number $=10$ data.

Multiplicity: Location of the I_number $=9$ data.

Reserved: Two slots per particle are reserved for future use.

\section{Kinematics type 4.}

This type of reaction starts with a 2-body reaction with an unstable intermediate nucleus. The data for the initial reaction is the same as for kinematics type 1 and is given in center-of-mass coordinates. Data for the secondary particles is given in laboratory coordinates.

Directory length: The length of this kinematics directory.

Identification of secondary particle: Identify the secondary particle by yo number.

Angular bins: Location of the I number $=1$ data.

Energy deposition: Location of the $I$ number $=10$ data.

Reserved locations: Two slots reserved for future use.

For each remaining particle: A block with the following segments. (The number of particles is given in the reaction directory.)

Identification of secondary particle: Identify the secondary particle by yo number.

Energy bins: Location of the I_number $=4$ data.

Angular bins: Location of the I_number $=1$ data if it is present in the file. Otherwise, these four numbers are zero.

Energy deposition: Location of the I_number = 10 data. 
Multiplicity: Location of the I_number $=9$ data.

Reserved: Two slots per particle are reserved for future use.

The unstable intermediary: Identify the unstable intermediary by its ZA number, $1000 * Z+A$.

Its mass: The atomic mass of the unstable intermediary.

\section{Kinematics type 5.}

This special kinematics type applies only to the cluster reaction $d\left(n, n^{\prime}\right) n p$.

Directory length: The length of this kinematics directory.

For each particle: A block with the following segments.

Identification of secondary particle: Identify the secondary particle by yo number.

Energy bins: Location of the I_number $=4$ data.

Angular bins: Location of the $I$ number $=1$ data if it is present in the file. Otherwise, these four numbers are zero.

Energy deposition: Location of the I number $=10$ data.

Multiplicity: Give the multiplicity of the secondary particle.

Reserved: Two slots per particle are reserved for future use.

\section{Kinematics type 6 and 7.}

These two kinematics types refer, respectively, to coherent and incoherent scattering of a photon by an electron. The corresponding kinematics directories take the same form.

Directory length: The length of this kinematics directory.

Photon scattering: For kinematics type 6 this segment gives the location of the I_number $=941$ data, and for type 7 it locates the I.number $=$ 942 data. 
Identification of secondary particle: Identify the secondary particle by yo number ( 7 for gamma).

Scatterer identification: Identify the scatterer (an electron.)

Energy deposition: Location of the I_number $=10$ data.

Reserved: Two slots per particle are reserved for future use.

\section{Reaction directories.}

Each reaction directory contains 22 items, plus a list of the secondary particles and their atomic masses. The specific items are as follows. The data locations are again relative to the start of the data for this target, and this reference point is given in the target directory.

1: The length of the reaction directory (22 plus 2 times the number of secondary particles).

2: The date of the latest modification, with 10 March 1993 coded as 930310.

3: The $\mathrm{C}$ reaction identifier.

4: The $S$ reaction switch, to tell the meaning of the variable $x 1$, item 13 on this list.

5: The atomic number and mass number of the target, coded as $1000 * Z+A$.

6: The number of entries in the cross-section table (from I_number $=0$ ).

7: 'The location of the cross-section table. The cross-section data is ordered by increasing energy.

8: The index of the energy bin for the first nonzero cross section. This amounts to an energy threshold.

9: The index of the energy bin for the last nonzero cross section. This amounts to a maximum energy.

10: The location of the data on energy produced (also from I_number $=0$ ). The number of entries is the same as the number of cross sections, and it is given in item 6 . 
11: Unused.

12: The energy of the reaction $q 0$ in Mev, computed from the mass balance.

13: The parameter $x 1$, with meaning specified by the switch $S$ given in item 4 above. This is commonly the excitation level.

14: The number of products, not including photons, but including residual nucleus when that is appropriate.

15: The location in the reaction directory of the list of product atomic numbers and mass numbers $1000 * Z+A$, relative to the start of the first reaction directory for this target.

16: The location in the reaction directory of the list of product atomic weights, relative to the start of the first reaction directory for this target.

17: The number of additional offsets. This number is currently one, to provide a slot for the location of energy production data for production cross section reactions $(C=5)$.

18: The location of the additional offsets, relative to the start of the first reaction directory for this target.

19: The kinematics type. This is one of the numbers $0,1, \ldots 7$, and its significance is explained in the description of the kinematics directories given above.

20: The location of the kinematics directory for this reaction, relative to the start of the target directory.

21: The length of the kinematics directory for this reaction.

22: The number of secondary particles for this reaction, including photons and not including a residual nucleus.

Particles: What follows is a list of the numbers $1000 * Z+A$ for the secondary particles produced by the reaction, including the residual nucleus but not photons. The location of this list is also given by item 15 above. Multiple particles are listed repeatedly, according to their number. 
Atomic masses: The particle list is followed by a list of their atomic masses, and the location of the first atomic mass is given by item 16 above.

Additional descriptors: Finally, we have the location of the energy production data for the $\mathrm{C}=5$ reaction. For other reactions this entry is zero.

\section{Target directory.}

The purpose of the target directory is to identify the target and to locate the reaction directories. The target directory is laid out as follows.

1: The length of the block containing this target directory, along with its associated reaction and kinematics directories.

2: The offset of the target data, relative to the start of the file. (This offset is the reference point for all of the data locations given in the reaction and kinematics directories.)

3: The length of the target data.

4: Three less than the length of the target directory.

5: The atomic number and mass number of the target, coded as $1000 * Z+A$.

6: The excitation energy in Mev.

7: The library date, with 11 July 1995 coded as 950711.

8: The number of reactions. Note that there may be data for the same kind of reaction with the residual at several different excitation levels, in which case these are counted as separate reactions.

9: Identify the library source. At the moment this string is just 4 blank spaces.

Reaction directory locations: For each reaction there follows two numbers, the length of the reaction directory and its location relative to the start of this target directory.

Reserved: Two slots are reserved for future use. 


\section{Overall directory}

The overall directory starts with 23 entries giving basic information about the file. Then comes information about the possible secondary particles. The energy groups are given next, followed by the the target temperature and a list of physical constants. The overall directory ends with the identification and location of each target directory. The specific content of the overall directory is as follows.

0: An 'mcf.bin' file always has a zero as the first entry. It is therefore convenient to start the count so that item number 1 is the first nonzero entry.

1: The length of the overall directory.

2: Identification of the incident particle. See the section on 'The code for particles' in the chapter Various identifiers.

3: Atomic number and mass number for the incident particle; coded as $1000 *$ $Z+A$.

4: The atomic mass of the incident particle.

5: The halflife of the incident particle. Stable particles are given a halflife of $10^{50}$.

6: The number of targets in this file.

7: The date of the library. Here, 21 November 1994 is coded as 941121.

8: The number of energy group bounds.

9: The location in this directory of the energy group bounds.

10: The number of temperatures. This number must be 1 , because Doppler broadening is not done in mcfgen.

11: The location in this directory of the temperatures.

12: The number of physical constants.

13: The location in this directory of the physical constants. 
14: The maximum size of a block of target data.

15: The interpolation type. This is 0 for linear-linear interpolation and 5 for $\log -\log$. But the use of linear-linear interpolation on $\log -\log$ data will introduce errors of no more than $0.1 \%$.

16: The length of a structure for identifying and locating a target directory. This number is currently 5 .

17: The location in this directory of the first structure for identifying and locating a target directory.

18: The number of additional directory items. In fact, this number is 22 , and these items identify the possible secondary particles.

19: The location of these additional directory items.

20: The amount of space in this directory devoted to structures identifying and locating target directories. It is the product of items 6 and 16.

21: A length once associated with target directories. Its value is 6 , but it is not used.

22: The library type. This is 0 for bin-averaged data and 1 for grid-based data.

Next comes the 22 additional items referred to in items 18 and 19 above.

Number of possible secondary particles: This number is now 7 .

The particle identifiers: The numbers 1-7 for neutron, proton, deuteron, triton. ${ }^{3} \mathrm{He}$, alpha, and photon.

Atomic masses: The next seven numbers are the atomic masses of the above particles.

ZA numbers: There follows the seven values of $1000 * Z+A$ for the possible secondary particles. 
Next comes the energy groups as mentioned in items 8 and 9 above. These are followed by the target temperature, and this location is given in item 11 in the overall directory. Then come the physical constants referred to in items 12 and 13. These constants are copied from the file

$$
\text { /nds/processing/constants/physcons }
$$

on the Nuclear Data Groups' network, and they are defined in that file. At the present time these constants are

1. A conversion factor, erg/Mev.

2. Avogadro's number.

3. Conversion factor, $\sec ^{* *} 2 / \mathrm{sh}^{* *} 2$.

4. Conversion factor, $(\mathrm{cm} / \mathrm{sh}) * \sqrt{\mathrm{amu} / \mathrm{Mev}}$, may be used to convert particle energy to velocity.

5. Conversion factor, Mev/amu.

6. Conversion factor, jerks $/ \mathrm{kton}$, where $1 \mathrm{kton}=10^{12} \mathrm{cal}$.

7. Conversion factor, Mev/jerk, where 1 jerk $=10^{16}$ ergs or $10^{9}$ joules.

8. Speed of light in vacuum in units of $\mathrm{cm} / \mathrm{sh}$.

9. Planck's constant in units of Mev sh.

10. Boltzmann's constant in units of $\mathrm{Mev} / \mathrm{K}$.

11. Rest mass of the electron in amu.

: The overall directory ends with a collection of the following structures, one for each target.

Target ZA: The atomic number and mass number of the target, coded as $1000 * Z+A$.

Target Z: The atomic number of the target.

Atomic mass: The atomic mass of the target.

Target directory location: The location of the target directory in this file.

Target directory length: The length of the target directory. 


\section{Code details for mcfgen}

The remainder of this document provides information to aid the programmer in maintenance of the mcfgen code. We begin with a section on data structures and then give some mathematical details on the calculation of equiprobable bins and on making log-log data suitable for linear-linear interpolation.

\section{Structures identifying the data}

The properties and locations of the data are given in the following structures: ident_data, i_react_data, and r_react_data. The source of the information for these structures is the array nhead/xhead, which is described in the section on the 'library' file. Each of the arrays ident_data, i_react_data, and r_react_data is declared in the file 'extcor.h', and they are filled by the subroutine rct. Their content is as follows.

\section{The array i_react_data}

This array contains the integer information about a target. The dimensions of the array are i_react_data(NUM_I_REACT, MAXC), with parameters WOM_I REACT $=7$ and MAXC $=200$. This array is reset for each new target. Its second index ranges over the reactions $\mathrm{C}$ for the target, and for each reaction the first index identifies the following items.

i react_data(1, c_count): The reaction identifier $\mathrm{C}$. See the section on reaction identifiers.

i_react_data (2, c_count): The number of I_number blocks (reaction properties) there are in the array ident_data for this reaction.

i_react_data ( 3, c_count): The date for the library data for this reaction.

i_react_data (4, c_count): The second reaction identifier $\mathrm{S}$. See the section on reaction identifiers. 
i_react_data(5, c_count): The index igsrt of the first nonzero average cross section. This amounts to an energy threshold.

i_react_data ( 6, c_count): The index igend of the last nonzero average cross section. This amounts to a maximum energy for the reaction.

i_react_data ( $7, c_{-}$count): The location for this reaction block in the array ident_data.

\section{The array r_react_data}

This array contains the real-number information about a target. The dimensions of the array are rreact_data(NUM_R_REACT, MAXC), with the values NUM_R_REACT $=6$ and MAXC $=200$. This array is reset for each new target. Its second index ranges over the reactions $\mathrm{C}$ for the target, and for each reaction the first index identifies the following items.

r_react_data (1, c_count): The energy created by the reaction, q0. For $\mathrm{C}=5$ this value is zero, and an array of flux-weighted bin averages $Q_{g}$ is provided.

r_react_data (2, c_count): The first extra information, $x 1$. See the section on the $\mathrm{S}$ reaction identifier for the content of this variable.

r_react_data( $3, c_{-}$count): The second extra information, $x 2$. The meaning of this variable is also determined by the value of the $S$ reaction identifier.

r_react_data (4, c_count): The calculated reaction threshold. This number is calculated by the subroutine parts and is based on the mass balance. It is not used now, but it is available.

r_react_data(5, c_count): The minimum energy for the reaction Emin. This number is printed out only for gamma production reactions.

r_react_data (6, c_count): The maximum energy for the reaction Emax. This number is also printed out only for gamma production reactions. 


\section{The array ident_data}

This array gives the basic information for each block of data for each reaction for a target. The dimensions of the array are ident_data(NUM_IDENT, MAXIC), with parameters NUM_IDENT $=4$ and MAXC $=400$. This array is reset for each new target. Its second index ranges over the reactions $\mathrm{C}$ and over the types of data Inumber for the target. For each reaction and for each value of I number the first index identifies the following items.

ident_data(1, I_C_count): The identifier I_number for the type of data. See the chapter on identifiers.

ident_data(2, I_C_count): Identifier yo for the first outgoing particle. See the chapter on identifiers.

ident_data(3, I_C_count): The data offset iifwa. Specifically, the location of this data block is given in nhead (iifwa). (The structure of nhead is defined in the the Target directory section of the chapter on the 'library' file).

ident_data (4, I_C_count): The interpolation type to be used on the data. The values used are 0 for linear-linear interpolation and 5 for $\log$-log interpolation. Note, however, that errors will be no worse than $0.1 \%$ if one uses linear-linear interpolation wnere $\log -\log$ is specified. 


\section{Equiprobable bins}

For pairs of values $(x, P(x))$ with $P$ a probability density, the routine eqpb uses linear interpolation to compute equi_dist, an equidistributed probability density. There are two basic applications of this routine: angular distributions (I_number $=1$ ) data and distributions of energy carried off by secondary particles ( $I$ number $=4$ data). But there is also the I_number $=3$ data for correlated distributions of angle and energy. We begin with a description of the general method and discuss later the special provisions made for the correlated distributions for I number $=3$.

The method used is as follows. Suppose that we want nbins bins, each with probability $1 /$ nbins, with the bottom of the lowest bin and the top of the highest bin fixed by the lowest and highest input values of $x$. The equidistributed interior bin boundaries are located at the $x$-values for which the accumulated probabilities are $k /$ nbins for $k=1,2, \ldots$, nbins -1 . The geometry of the problem is that we want the set of $x$ points which give equal area under the graph of $P$. From a mathematical point of view, what we want is the points $\xi_{k}$ (for $k=0,1, \ldots$, nbins) such that $\xi_{0}$ is the first library $x$-value and

$$
\int_{\xi_{0}}^{\xi_{k}} P(x) d x=\frac{k}{\text { nbins }} .
$$

But $P$ is piecewise linear, so this condition requires us to add up areas of trapezoids.

Suppose that we seek the $k$-th equidistribution point, $\xi_{k}$. We start by adding the areas of the trapezoids given by the library $(x, P(x))$ data, and we eventually find the first time that the accumulated sum exceeds the desired area $k /$ nbins. This brackets $\xi_{k}$ between two of the library $x$-values, and $P$ is linear on this interval. The integral of $P$ is therefore quadratic on the interval, and the determination of $\xi_{k}$ requires the solution of a quadratic equation. Only one root of the quadratic makes physical sense, and the algorithm takes advantage of this fact. The extraneous root is easily understood by looking at the Fig. 1. In that figure the piecewise-linear library data is plotted as a broken line, and the straight-line section of interest to us is extrapolated as a dashed line. Because of the congruent triangles between $B$ and $C$, it is clear that

$$
\int_{A}^{B} P(x) d x=\int_{A}^{C} P(x) d x .
$$




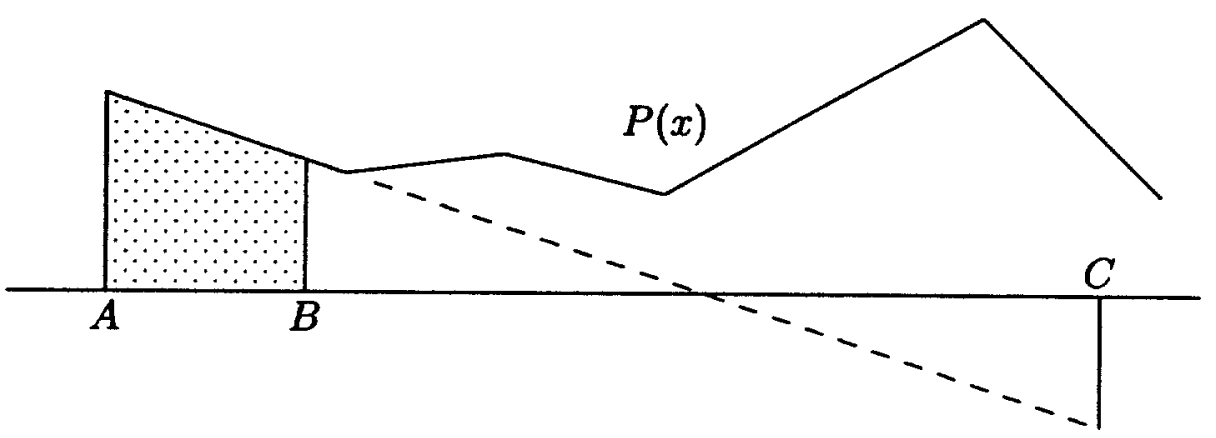

Figure 1: Only one root is physical.

That is, the quadratic equation for the equidistribution point has two roots, $B$ and $C$, but the root $C$ is physically impossible. 


\section{Correlated angle-energy equidistribution}

The algorithm to produce equidistrubuted correlated angle-energy bins from I number $=3$ data is implemented by the routine iequ3. The data for both the angle-energy probabilities (I_number $=3$ ) and for the angular probabilities ( $I$ _number $=1$ ) must be present. Furthermore, the lists of incident energies at which the I_number $=3$ and I_number $=1$ probabilities are given must be identical.

The computation of equiprobable angle-energy is done in three steps. First, the Inumber $=1$ data is used to make an equidistribution of the cosines using the method described above. For each of these equidistributed cosines we need to create equidistributed secondary energy bins. So, the second step is the creation of secondary energy distributions at these new cosines. The third step is the equidistribution of these energies. Only the second step is new, and its details are as follows.

Consider one of the equidistributed cosines. We bracket it between two I_number $=3$ (or equivalently, I_number $=1$ ) cosines from the ' 1 ibrary' file. We want to use the (energy, probability density) data from these bracketing cosines to produce (energy, probability density) pairs for the new cosine. This is illustrated in the Fig. 2, which has secondary energy increasing to the right and cosine increasing upward. The new equidistributed cosine $C_{\text {new }}$ lies between the library cosines $C_{0}$ and $C_{1}$. We want probablility densities for $C_{\text {new }}$, starting from probability densities at one set of energies for $C_{0}$ (denoted by dots) and at another set of energies for $C_{1}$ (denoted by crosses). The algorithm is based on multiple linear interpolation as follows.

1.. We start by translating and scaling the secondary energy ranges for the cosines $C_{0}$ and $C_{1}$ to the interval $0 \leq E \leq 1$, and we scale the probability densities correspondingly. The figure shows the situation after this scaling. There are several reasons for starting with this scaling. For one thing, it ensures proper linear dependence on incident energy for the minimum and maximum energy of the secondary particle. For another, it leads to more accurate interpolation of such physical features as resonance peaks. Finally, it makes the interpolation process clearer.

2. We merge these scaled energy lists to produce a master secondary energy list, denoted by the vertical lines in the figure. 


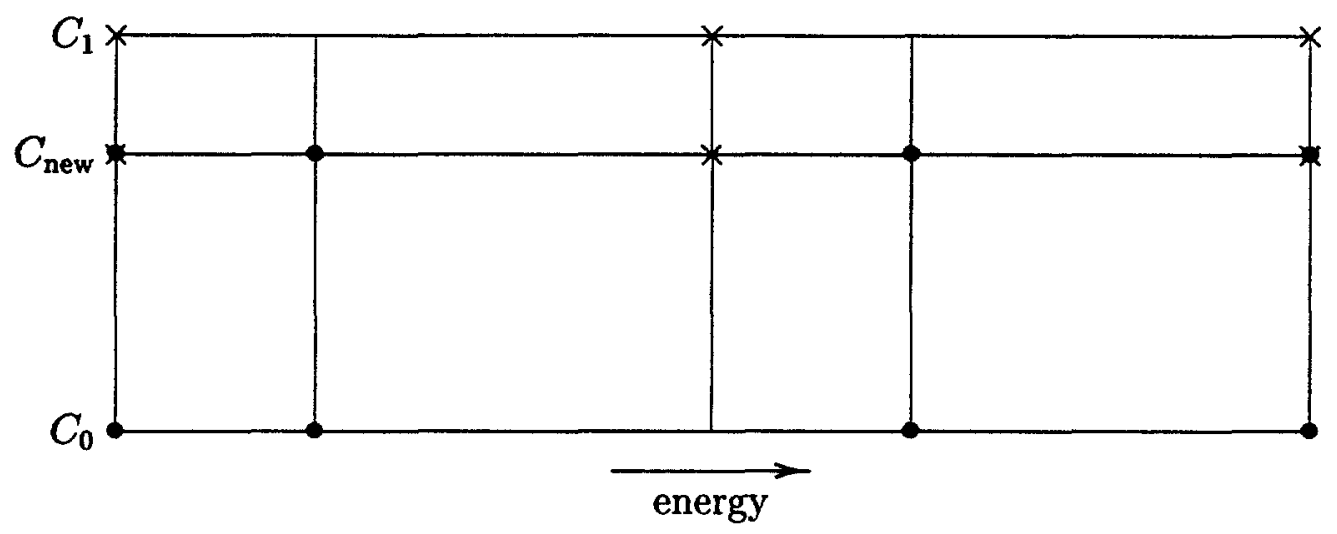

Figure 2: Double interpolation of probability density.

3. For each library cosine $C_{0}$ and $C_{1}$ we interpolate in energy to get probability densities at the full merged list of secondary energies. This is interpolation horizontally in Fig. 2.

4. Then at each secondary energy we interpolate vertically in the figure, to get the probability densities at the equiprobable cosine $C_{\text {new }}$.

5. Now that we have probability densities, we use the method described in the previous section to get equiprobable secondary energy bins at $C_{\text {new }}$, but these are still scaled to $0 \leq E \leq 1$.

6. Finally, we undo the scaling by mapping from $0 \leq E \leq 1$ to the physical $:$ energy interval. 


\section{Log-log interpolation}

This section explains how mcfgen fills in data points to permit linear-linear interpolation of log-log data. This fill-in is done by the subroutine thicken, and it applies only when the incident particle is a photon.

The input to this subroutine consists of data representable as a line on a $\log -\log$ plot, together with a relative error tolerance $\epsilon$. The output is a possibly denser data set, for which linear interpolation agrees with log-log interpolation to within a relative error $\epsilon$. In mcfgen the value of $\epsilon$ is chosen to ensure an interpolation error of no more than $0.1 \%$.

\section{The interpolation problem.}

Suppose that we are given two data points $\left(x_{0}, u_{0}\right)$ and $\left(x_{1}, u_{1}\right)$, and assume that between these two points $u$ is representable as a line on a log-log plot. That is, we have

$$
\log u=\log u_{0}+\alpha\left(\log x-\log x_{0}\right)
$$

with

$$
\alpha=\frac{\log \left(u_{1} / u_{0}\right)}{\log \left(x_{1} / x_{0}\right)} .
$$

Clearly, we must have $x_{0}, u_{0}, x_{1}$, and $u_{1}$ all positive. We also assume that $x_{0}<x_{1}$. Note that we may write (1) in the form

$$
u=u_{0}\left(\frac{x}{x_{0}}\right)^{\alpha}
$$

for $x_{0} \leq x \leq x_{1}$. Our problem is that for a prescribed error tolerance $\epsilon$, we are to construct a piecewise-linear approximation to $u$ for which the relative error is never larger than $\epsilon$.

The most efficient broken-line approximation to the graph of (3) would cross the curve twice on each linear segment. Instead of doing that, however, the code puts the corner points of the broken line exactly on the graph of $u$. The broken line therefore lies on one side of the graph of (3), and we insert about twice as many corner points as there would be in the best piecewiselinear approximant. 


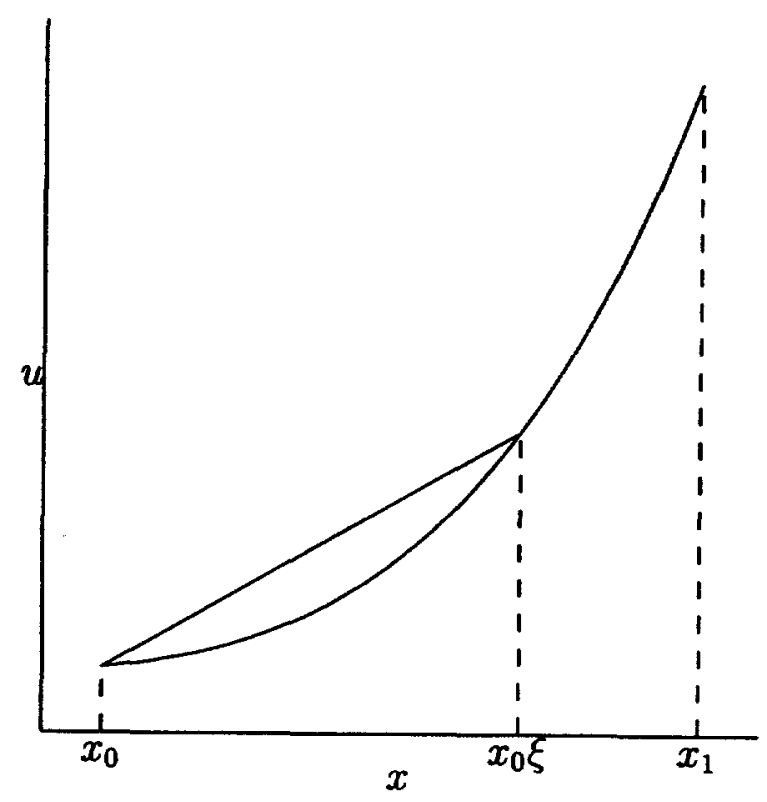

Figure 3: Linear interpolation.

The analysis proceeds as follows. Observe that the second derivative of $u$ is

$$
\frac{d^{2} u}{d x^{2}}=\alpha(\alpha-1) \frac{u_{0}}{x_{0}^{2}}\left(\frac{x}{x_{0}}\right)^{\alpha-2}
$$

so that $u$ is convex if $\alpha 1$ or $\alpha<0$ and concave if $0<\alpha<1$. Consequently, the line from $\left(x_{0}, u_{0}\right)$ to $\left(x_{1}, u_{1}\right)$ lies above the graph of $u$ if $\alpha>1$ or $\alpha<0$ and below it if $0<\alpha<1$. A case with $d^{2} u / d x^{2}>0$ is illustrated in Figure 3 .

\section{The accuracy of linear interpolation.}

It turns out to be convenient to label the first insertion point as $x_{0} \xi$, and we shall use linear interpolation for $x_{0}<x<x_{0} \xi$. We therefore want $x_{0}<x_{0} \xi \leq x_{1}$, or in other words, $1<\xi \leq x_{1} / x_{0}$. (If we decide that $x_{0} \xi=x_{1}$, then we don't insert any new points.) The value of $u$ corresponding to $x=x_{0} \xi$ is taken as given by (3), $u=u_{0} \xi^{\alpha}$. The line interpolating between the two points $\left(x_{0}, u_{0}\right)$ and $\left(x_{0} \xi, u_{0} \xi^{\alpha}\right)$ is

$$
v=u_{0}\left[1+\beta\left(\frac{x}{x_{0}}-1\right)\right]
$$


with

$$
\beta=\frac{\left(\xi^{\alpha}-1\right)}{(\xi-1)} .
$$

See Figure 3. Our object is to select $\xi$ in such a way that the relative error

$$
E=\frac{v-u}{u}
$$

satisfies $|E| \leq \epsilon$ for $x_{0}<x<x_{0} \xi$. It is important to observe that $u$ and $v$, and consequently the relative error $E$ depend on $x$ only in the combination $x / x_{0}$.

The largest absolute relative error occurs at the point $\widetilde{x}$ where $d E / d x=0$. By (7) this is the point at which

$$
u \frac{d v}{d x}=v \frac{d u}{d x}
$$

It follows from (3) and (5) that this occurs where

$$
\frac{\beta u_{0} u}{x_{0}}=\frac{\alpha u v}{x}
$$

This is equivalent to

$$
\beta u_{0} \frac{x}{x_{0}}=\alpha v .
$$

Let us label the solution of $(10)$ by the notation $\widetilde{x}$. Upon substituting the definition (5) of $v$ into (10) and solving for $\widetilde{x}$, we find that the largest absolute relative error occurs at

$$
\frac{\widetilde{x}}{x_{0}}=\frac{\alpha(\beta-1)}{\beta(\alpha-1)}
$$

Insertion of the definition (6) of $\beta$ shows that

$$
\frac{\tilde{x}}{x_{0}}=\frac{\alpha\left(\xi^{\alpha}-\xi\right)}{(\alpha-1)\left(\xi^{\alpha}-1\right)}
$$

\section{Singularities.}

Note that the right-hand side of (12) takes the form $0 / 0$ in the special cases $\alpha=0$ and $\alpha=1$. That is no great surprise, since (3) reduces to $u=u_{0}$ for $\alpha=0$ and to $u=u_{0} x / x_{0}$ for $\alpha=1$, both being equations for which 
linear interpolation is exact. Consequently, $E=0$ identically if $\alpha=0$ or $\alpha=1$, and we don't need to insert any points in those cases. We do need to be concerned, however, about nearby cases in which numerical evaluation of (12) would lead to severe loss of accuracy through subtraction of nearly equal numbers. We use l'Hospital's rule to show that these singularities are removable, and we find the constant term of the Taylor series for (12) expanded about $\alpha=0$ and $\alpha=1$.

For $\alpha \approx 0$ the singular behavior is due to the factor $\alpha /\left(\xi^{\alpha}-1\right)$. We therefore use l'Hospital's rule to show that

$$
\frac{\alpha}{\xi^{\alpha}-1} \approx \frac{1}{\xi^{\alpha} \log \xi} \approx \frac{1}{\log \xi}
$$

as $\alpha \rightarrow 0$. The remaining factor $\left(\xi^{\alpha}-\xi\right) /(\alpha-1)$ has the limiting value

$$
\lim _{\alpha \rightarrow 0} \frac{\xi^{\alpha}-\xi}{\alpha-1}=\xi-1 .
$$

Thus, we conclude that for $\alpha \approx 0$ we should replace (12) by

$$
\frac{\widetilde{x}}{x_{0}} \approx \frac{\xi-1}{\log \xi} .
$$

Similarly, for $\alpha \approx 1$ it follows from l'Hospital's rule that

$$
\frac{\xi^{\alpha}-\xi}{\alpha-1} \approx \xi^{\alpha} \log \xi \approx \xi \log \xi .
$$

For $\alpha \approx 1$ the remaining factor is

$$
\frac{\alpha}{\xi^{\alpha}-1} \approx \frac{1}{\xi-1} .
$$

Consequently, for $\alpha \approx 1$ we replace (12) by

$$
\frac{\tilde{x}}{x_{0}} \approx \frac{\xi \log \xi}{\xi-1} .
$$




\section{Test whether points need to be inserted.}

Now that the theoretical background has been presented, we turn to the numerical algorithm. The first step is to to test whether additional points need to be inserted into the interval $x_{0}<x<x_{1}$. This is done in the following steps.

1. Set $\xi=x_{1} / x_{0}$.

2. Compute $\tilde{x}$ by formula (12), (15), or (18), depending on the value of $\alpha$.

3. Compute

$$
\widetilde{u}=u_{0}\left(\frac{\widetilde{x}}{x_{0}}\right)^{\alpha}
$$

and (with $\beta$ as defined by (6))

$$
\tilde{v}=u_{0}\left[1+\beta\left(\frac{\tilde{x}}{x_{0}}-1\right)\right] .
$$

The largest relative error is

$$
|\max E|=\left|\frac{\tilde{v}-\tilde{u}}{\tilde{u}}\right| .
$$

4. If $|\max E| \leq \epsilon$, we are finished. Otherwise, we must insert additional points for the linear interpolation.

\section{The first insertion point.}

We give here one way to specify the first insertion point. The method actually used in the code is a little bit different, and it is given in the next section.

If we need to insert additional points, the first such insertion point is determined by the value of $\xi$ with the following properties: (i) $x_{0}<x_{0} \xi<x_{1}$ and (ii) upon substituting $\xi$ into (12), (15), or (18) (depending on the value of $\alpha$ ) and computing $\max E$ by (19-21) we get $|\max E|=\epsilon$. The best way to find $\xi$ is to use zeroin, the standard routine for finding the root of an equation. Note that we have two starting values which bracket the solution: (i) The guess $\xi=1$ is too small because it gives $\max E=0$, and (ii) we have 
already computed $\max E$ for $\xi=x_{1} / x_{0}$ and found this value of $\xi$ to be too large. In fact, there is an even better initial guess, $\xi=1+\delta$ with

$$
\delta=\sqrt{\frac{8 \epsilon}{|\alpha(\alpha-1)|}}
$$

which may be obtained from a power series expansion of $\max E$. The relation (22) is derived at the end of this section.

Once we have found the value of $\xi$ which gives $|\max E|=\epsilon$, we take $x_{0} \xi$ as our first insertion point, and we set its $u$-value to $u_{0} \xi^{\alpha}$ in accordance with (3).

\section{Subsequent insertion points.}

We could now repeat the preceding algorithm with $x_{0} \xi$ as the new left-hand end point, but there is an easier way. Because $x$ appears in our formulas only in the ratio $x / x_{0}$, we already know the result. It is that if $x_{0} \xi^{2}>x_{1}$, we don't need a second insertion point. Otherwise, the second insertion point is $x_{0} \xi^{2}$ and its $u$-value is $u_{0} \xi^{2 \alpha}$.

By repeating this argument, we see that in order to determine the number of insertion points necessary, we find the smallest integer $N$ such that

$$
\xi^{N} \geq \frac{x_{1}}{x_{0}}
$$

Then we could use as the inserted points and their $u$-values

$$
\left(x_{0} \xi, u_{0} \xi^{\alpha}\right),\left(x_{0} \xi^{2}, u_{0} \xi^{2 \alpha}\right), \cdots,\left(x_{0} \xi^{N-1}, u_{0} \xi^{(N-1) \alpha}\right) .
$$

The only reason we don't use the collection (24) is that the final interval $x_{0} \xi^{N-1}<x<x_{1}$ could be very short.

What we actually do is as follows. We compute the smallest integer $N$ such that (23) holds, and we set

$$
\eta=\left(\frac{x_{1}}{x_{0}}\right)^{1 / N}
$$

We then use insertion points and $u$-values given by

$$
\left(x_{0} \eta, u_{0} \eta^{\alpha}\right),\left(x_{0} \eta^{2}, u_{0} \eta^{2 \alpha}\right), \cdots,\left(x_{0} \eta^{N-1}, u_{0} \eta^{(N-1) \alpha}\right) .
$$

The collection (26) gives a uniform subdivision with respect to $\log x$. 


\section{An approximation for the first insertion point.}

Let us now derive the approximation (22) for the first insertion point $\xi=$ $1+\delta$. This is obtained by substituting the first few tems of the power series expansion for $\max E$, defined in (21), into the equation $|\max E(\xi)|=\epsilon$.

It turns out that we need to to carry terms out to the cubic in

$$
\xi^{\alpha} \approx 1+\alpha \delta+\frac{\alpha(\alpha-1)}{2} \delta^{2}+\frac{\alpha(\alpha-1)(\alpha-2)}{6} \delta^{3} .
$$

Then it follows from (12) that the maximum relative error is located approximately at

$$
\frac{\tilde{x}}{x_{0}} \approx 1+\frac{\delta}{2}-\frac{\alpha+1}{12} \delta^{2} .
$$

Let us now determine the values of $u$ and the linear approximation $v$ at this approximate worst point. Substitution of (28) into (19) yields the approximation to $\tilde{u}$

$$
\widetilde{u} \approx u_{0}\left\{1+\frac{\alpha}{2} \delta+\frac{\alpha(\alpha-5)}{24} \delta^{2}\right\} .
$$

Similarly, we conclude from (20) and (6) that

$$
\tilde{v} \approx u_{0}\left\{1+\frac{\alpha}{2} \delta+\frac{\alpha(\alpha-2)}{6} \delta^{2}\right\} .
$$

If we insert (29-30) into the definition (21) of $\max E$, we find that

$$
\max E \approx \frac{\alpha(\alpha-1)}{8} \delta^{2} .
$$

The approximate solution (22) of the equation $|\max E|=\epsilon$ now follows directly from (31).

\section{Summary.}

We have shown how to insert points into the data so that linear-linear interpolation of $\log -\log$ data is accurate to within a relative error $\epsilon$. This is done by solving the equation $|\max E|=\epsilon$ for $\xi$, by taking $N$ such that (23) holds, and by inserting the points given by (25-26). We have also seen that a good initial approximation to $\xi$ is given by

$$
\xi=1+\delta
$$


with $\delta$ as in (22).

The original version of mcfgen computed $\max E$ by the formulas (19$21)$. It differed, though, in that it evaluated $|\max E|$ at the point $\eta$ given by (25), trying $N=1,2, \ldots$, consecutively and stopping the first time that $|\max E| \leq \epsilon$. Our approach using the root finder is more efficient as soon as $N>3$. 


\section{References}

1. D. E. Cullen, "TART95: A coupled neutron-photon Monte Carlo transport code", Report UCRL-MA-121319, Lawrence Livermore National Laboratory, July 1995.

2. R. J. Howerton, R. E. Dye, P. C. Giles, J. R. Kimlinger, S. T. Perkins, and E. F. Plechaty, "OMEGA: a Cray 1 executive code for LLNL nuclear data libraries", Report UCRL-50400, Vol. 25, Lawrence Livermore National Laboratory, August 1983.

3. R. J. Howerton, R. E. Dye, and S. T. Perkins, "Evaluated nuclear data libraries", Report UCRL-50400, Vol. 4, Rev. 1, Lawrence Livermore National Laboratory, October 1981.

4. S. T. Perkins, "Specification of the all-particle Monte Carlo data files, MCFYi", Draft Report, Lawrence Livermore National Laboratory, July 1994.

5. J. A. Rathkopf, "Format of binary ENDL-type libraries", Report \#PD166, Lawrence Livermore National Laboratory, 17 October 1988. 